\title{
Asymmetric Friedel-Crafts addition of indoles to $N$-sulfonyl aldimines: A simple approach to optically active 3 -indolylmethanamine derivatives
}

\author{
Yi-Xia Jia, Jian-Hua Xie, Hai-Feng Duan, Li-Xin Wang, Qi-Lin Zhou* \\ State Key Laboratory and Institute of Elemento-organic Chemistry, Nankai University Tianjin \\ 300071, China
}

CONTENTS :

(A) Preparation of Substrates $1 \mathrm{k}-\mathbf{q}$ And Their Analytical Data

(B) General Procedure for the Catalytic Asymmetric Friedel-Crafts Reaction And Analytical Data of the Products

(C) Crystal Data And Structure Refinement for (S)-2f

\section{General:}

All reactions and manipulations which are sensitive to moisture or air were performed in an argon-filled glove box (VAC DRI-LAB HE 493) or using standard Schlenk techniques. Commercial reagents were used as received without further purification unless otherwise noticed. Anhydrous $\mathrm{CH}_{2} \mathrm{Cl}_{2}$ and $\mathrm{CH}_{2} \mathrm{ClCH}_{2} \mathrm{Cl}_{2}$ were freshly distilled from calcium hydride. Substrates 1a-j were prepared according to the literature. ${ }^{1}$ Melting points were measured on a RY-I apparatus and uncorrected. NMR spectra were recorded with a Bruker spectrometer at $300 \mathrm{MHz}\left({ }^{1} \mathrm{H}\right.$ NMR), 75 $\mathrm{MHz}\left({ }^{13} \mathrm{C} \mathrm{NMR}\right)$. Chemical shifts were reported in ppm down field from internal $\mathrm{Me}_{4} \mathrm{Si}$. Optical rotations were determined using a Perkin Elmer Model 341 polarimeter. Elemental analyses were performed on Yanaca CDRDER MT-3 instrument. Mass spectra were recorded on a LCQ Advantage spectrometer with ESI resource. HRMS were recorded on VG ZAB-HS mass 
spectrometer with EI or ESI resource. HPLC analyses were performed using a Hewlett Packard Model HP 1100 Series or Waters 2996 chromatography. X-ray diffraction analysis was performed on a Bruker smart-1000 X-ray diffraction meter.

\section{(A) Preparation of Substrates $1 \mathrm{k}-\mathbf{q}$ And Their Analytical Data}

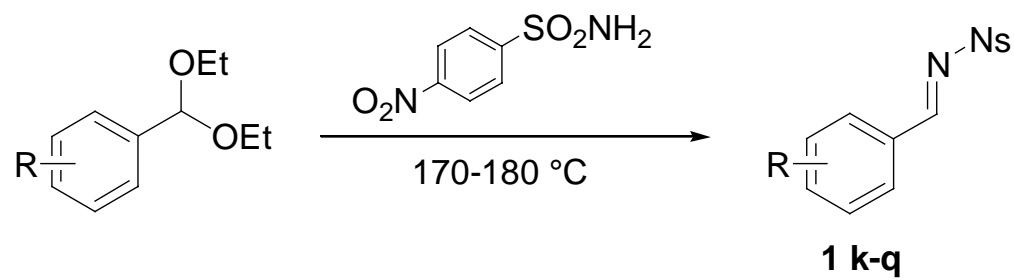

General procedure for preparation of substrates $1 \mathbf{k}-\mathbf{q}$ : A mixture of aromatic aldehyde diethyl $\operatorname{acetal}^{2}(0.12 \mathrm{~mol})$ and 4-nitrobenzenesulfonamide $(0.1 \mathrm{~mol})$ were heated at $170-180{ }^{\circ} \mathrm{C}$ under $\mathrm{N}_{2}$ atmosphere for 1-3 $\mathrm{h}$, and the formed $\mathrm{EtOH}$ was collected in a receiving flask. After cooling, the reaction mixture was dissolved in warm EtOAc, treated with $n$-pentane and allowed to stand at room temperature overnight. The crystal was collected by filtration, washed with $n$-pentane and dried.

$\boldsymbol{N}$-Benzylidene-4-nitrobenzenesulfonamide (1k) ${ }^{3}$ : 89\% yield, ${ }^{1} \mathrm{H}$ NMR $\left(300 \mathrm{MHz}, \mathrm{CDCl}_{3}\right)$ : $\delta 7.53(\mathrm{t}, J=7.8 \mathrm{~Hz}, 2 \mathrm{H}), 7.68(\mathrm{t}, J=7.8 \mathrm{~Hz}, 1 \mathrm{H}), 7.96(\mathrm{~d}, J=8.1 \mathrm{~Hz}, 2 \mathrm{H}), 8.22(\mathrm{~d}, J=8.7 \mathrm{~Hz}, 2 \mathrm{H})$, $8.40(\mathrm{~d}, J=8.7 \mathrm{~Hz}, 2 \mathrm{H}), 9.13(\mathrm{~s}, 1 \mathrm{H})$.

$N$-(4-Fluorobenzylidene)-4-nitrobenzenesulfonamide (11) ${ }^{3}: 92 \%$ yield, ${ }^{1} \mathrm{H}$ NMR $(300 \mathrm{MHz}$, $\left.\mathrm{CDCl}_{3}\right): \delta 7.22(\mathrm{~d}, J=8.4 \mathrm{~Hz}, 2 \mathrm{H}), 8.0(\mathrm{~d}, J=8.4 \mathrm{~Hz}, 1 \mathrm{H}), 8.22(\mathrm{~d}, J=9.0 \mathrm{~Hz}, 2 \mathrm{H}), 8.41(\mathrm{~d}, J=9.0$ $\mathrm{Hz}, 2 \mathrm{H}), 9.10(\mathrm{~s}, 1 \mathrm{H})$.

N-(4-Methylbenzylidene)-4-nitrobenzenesulfonamide (1m): $94 \%$ yield, pale yellow powder, mp: 200-202 ${ }^{\circ} \mathrm{C},{ }^{1} \mathrm{H}$ NMR (300 MHz, $\left.\mathrm{CDCl}_{3}\right): \delta 2.45$ (s, 3H), 7.33 (d, J=7.8 Hz, 2H), 7.85 (d, $J=$ $7.8 \mathrm{~Hz}, 2 \mathrm{H}), 8.21(\mathrm{~d}, J=8.7 \mathrm{~Hz}, 2 \mathrm{H}), 8.38(\mathrm{~d}, J=8.7 \mathrm{~Hz}, 2 \mathrm{H}), 9.08(\mathrm{~s}, 1 \mathrm{H}) ;{ }^{13} \mathrm{C} \mathrm{NMR}(75 \mathrm{MHz}$, $\left.\mathrm{CDCl}_{3}\right): \delta 22.1,124.3,129.3,129.5,130.2,131.8,144.5,147.5,150.5,172.1$; EI MS m/z 304; Anal. Calcd. for $\mathrm{C}_{14} \mathrm{H}_{12} \mathrm{~N}_{2} \mathrm{O}_{4} \mathrm{~S}$ : C, 55.25; H, 3.97; N, 9.21. Found: C, 55.54; H, 3.73; N, 9.56. 

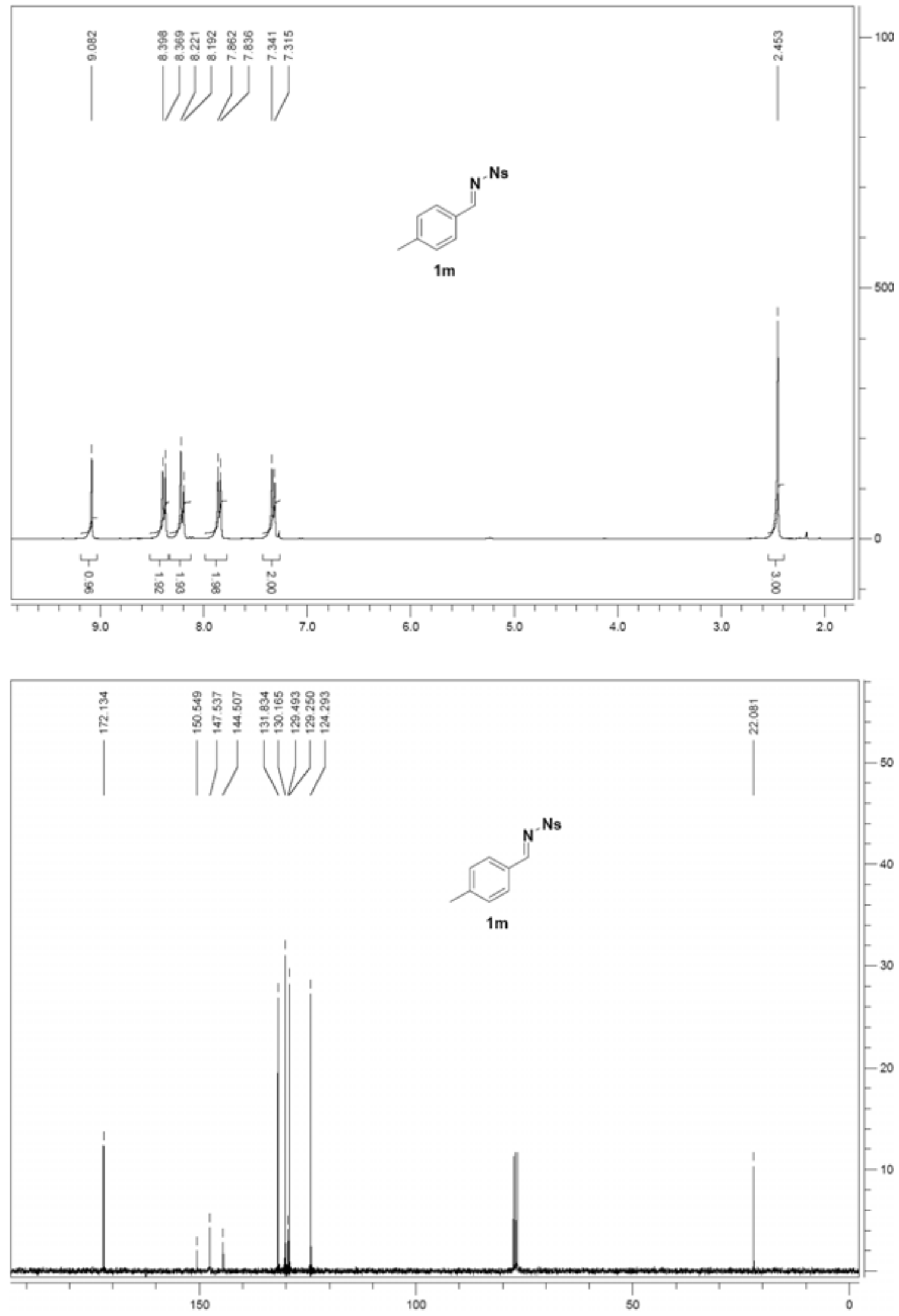

$N$-(3-Methylbenzylidene)-4-nitrobenzenesulfonamide (1n): 91\% yiled, pale yellow powder, mp: $122-124{ }^{\circ} \mathrm{C},{ }^{1} \mathrm{H}$ NMR $\left(300 \mathrm{MHz}, \mathrm{CDCl}_{3}\right): \delta 2.41(\mathrm{~s}, 3 \mathrm{H}), 7.38-7.49(\mathrm{~m}, 2 \mathrm{H}), 7.74(\mathrm{~d}, J=7.5$ $\mathrm{Hz}, 1 \mathrm{H}), 7.78(\mathrm{~s}, 1 \mathrm{H}), 8.21(\mathrm{~d}, J=9.0 \mathrm{~Hz}, 2 \mathrm{H}), 8.38(\mathrm{~d}, J=9.0 \mathrm{~Hz}, 2 \mathrm{H}), 9.09(\mathrm{~s}, 1 \mathrm{H}) ;{ }^{13} \mathrm{C}$ NMR $(75$ 
$\left.\mathrm{MHz}, \mathrm{CDCl}_{3}\right): \delta 21.1,124.3,129.2,129.3,129.4,131.7,131.9,136.7,139.4,144.3,150.6,172.6$; EI MS m/z 304; Anal. Calcd. for $\mathrm{C}_{14} \mathrm{H}_{12} \mathrm{~N}_{2} \mathrm{O}_{4} \mathrm{~S}: \mathrm{C}, 55.25 ; \mathrm{H}, 3.97 ; \mathrm{N}, 9.21$. Found: C, 55.45; H, 3.51; N, 9.27 .
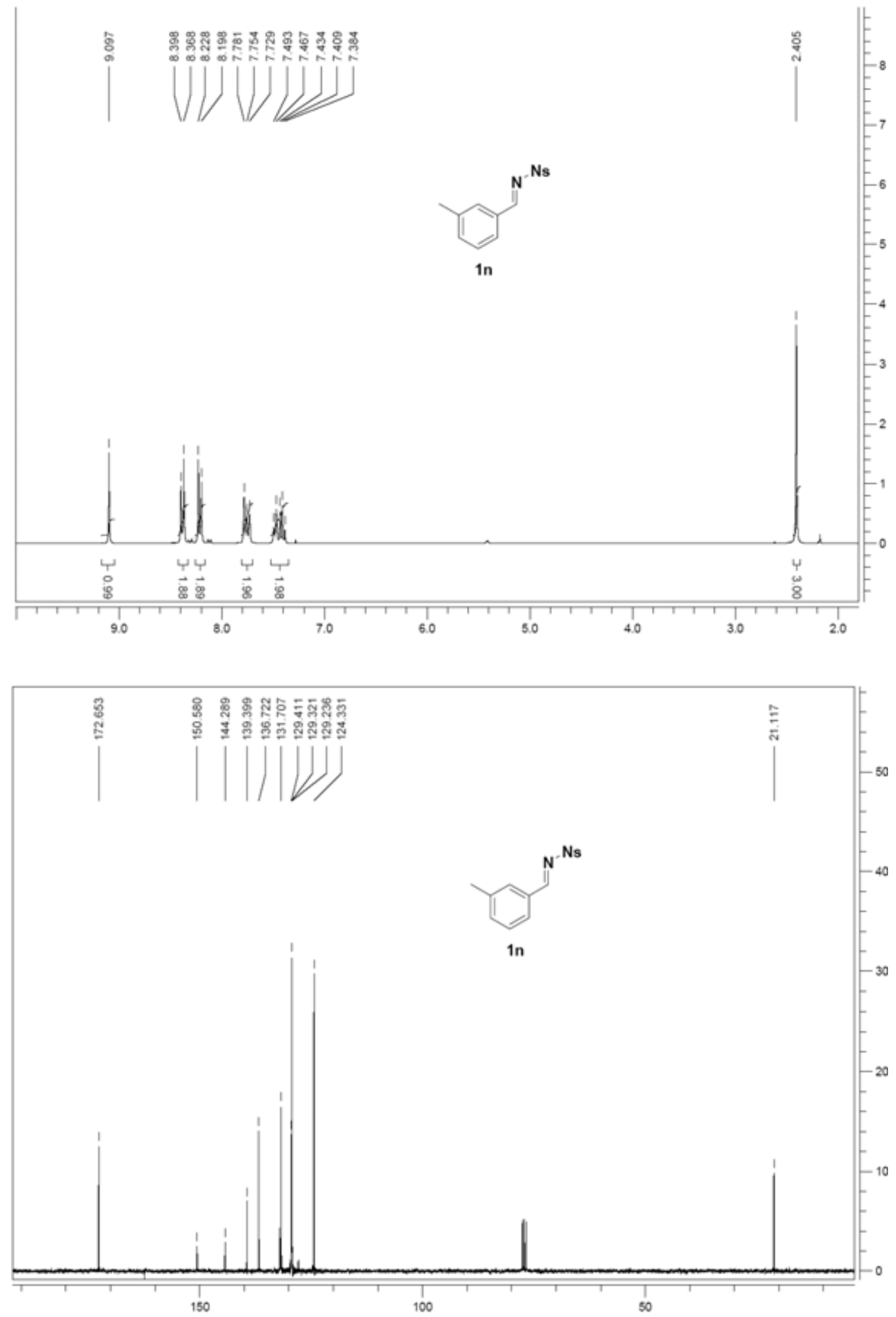
$N$-(2-Methylbenzylidene)-4-nitrobenzenesulfonamide (10): 94\% yield, pale yellow powder, mp: $159-161{ }^{\circ} \mathrm{C},{ }^{1} \mathrm{H}$ NMR $\left(300 \mathrm{MHz}, \mathrm{CDCl}_{3}\right): \delta 2.65(\mathrm{~s}, 3 \mathrm{H}), 7.32(\mathrm{t}, J=7.5 \mathrm{~Hz}, 2 \mathrm{H}), 7.53(\mathrm{t}, J=$ $7.5 \mathrm{~Hz}, 2 \mathrm{H}), 8.02(\mathrm{~d}, J=7.2 \mathrm{~Hz}, 1 \mathrm{H}), 8.22$ (d, J=8.7 Hz, 2H), 8.39 (d, J = 8.7 Hz, 2H), 9.43 (s, $1 \mathrm{H}) ;{ }^{13} \mathrm{C}$ NMR $\left(75 \mathrm{MHz}, \mathrm{CDCl}_{3}\right): \delta 19.7,124.3,126.8,129.3,130.1,131.1,131.8,135.4,142.9$, 144.5, 150.6, 170.9; EI MS m/z 304; Anal. Calcd. for $\mathrm{C}_{14} \mathrm{H}_{12} \mathrm{~N}_{2} \mathrm{O}_{4} \mathrm{~S}: \mathrm{C}, 55.25 ; \mathrm{H}, 3.97$; N, 9.21. Found: C, 55.09; H, 4.11; N, 9.00.
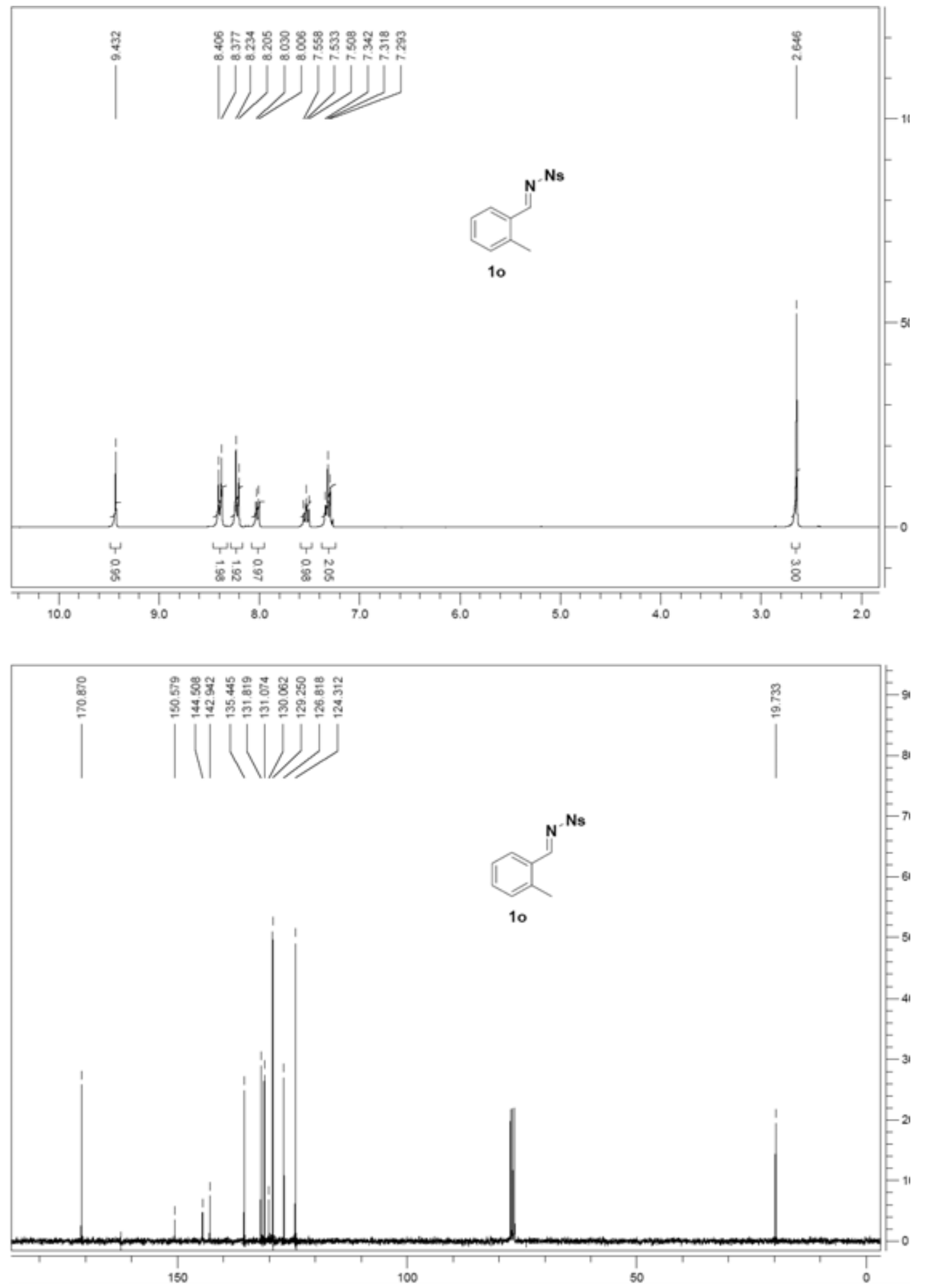
N-(4-Methoxybenzylidene)-4-nitrobenzenesulfonamide (1p): 87\% yield, pale yellow powder, mp: $175-177{ }^{\circ} \mathrm{C},{ }^{1} \mathrm{H}$ NMR $\left(300 \mathrm{MHz}, \mathrm{CDCl}_{3}\right): \delta 3.91(\mathrm{~s}, 3 \mathrm{H}), 7.0(\mathrm{~d}, J=8.4 \mathrm{~Hz}, 2 \mathrm{H}), 7.92(\mathrm{~d}, J=$ $8.4 \mathrm{~Hz}, 2 \mathrm{H}), 8.19$ (d, J=7.8 Hz, 2H), 8.37 (d, J=7.8 Hz, 2H), $9.02(\mathrm{~s}, 1 \mathrm{H}) ;{ }^{13} \mathrm{C}$ NMR $(75 \mathrm{MHz}$, $\left.\mathrm{CDCl}_{3}\right): \delta 55.8,114.9,124.2,124.8,129.1,134.2,144.8,150.4,166.1,171.2$; EI MS m/z 320;Anal. Calcd. for $\mathrm{C}_{14} \mathrm{H}_{12} \mathrm{~N}_{2} \mathrm{O}_{5} \mathrm{~S}$ : C, 52.49; H, 3.78; N, 8.75. Found: C, 52.90; H, 3.45; N, 8.65.
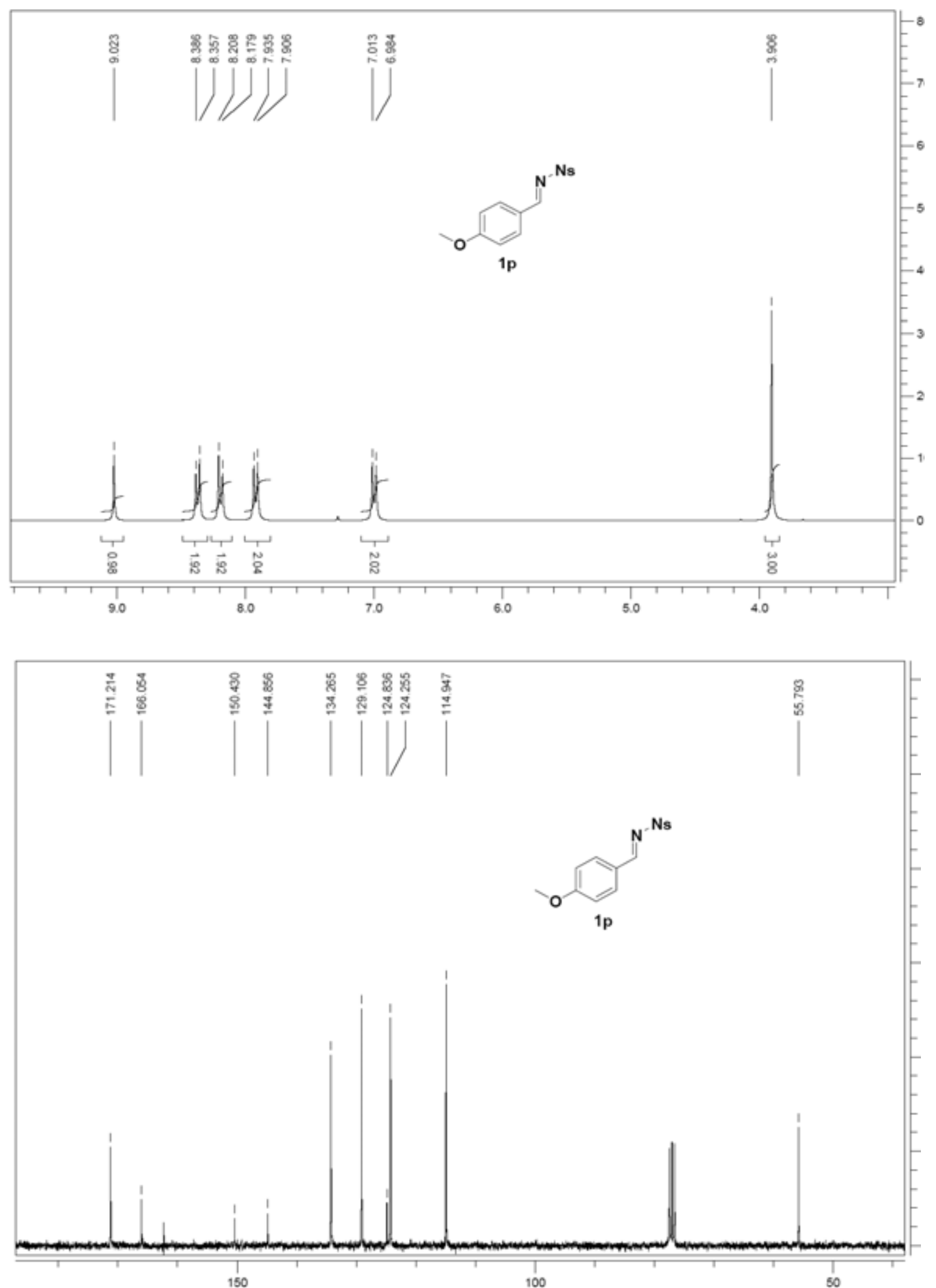
$N$-(1-Naphthylidene)-4-nitrobenzenesulfonamide (1q): 92\% yield, pale brown powder, mp: $179-181{ }^{\circ} \mathrm{C},{ }^{1} \mathrm{H}$ NMR $\left(300 \mathrm{MHz}, \mathrm{CDCl}_{3}\right): \delta 7.59-7.75(\mathrm{~m}, 3 \mathrm{H}), 7.96(\mathrm{~d}, J=7.8 \mathrm{~Hz}, 1 \mathrm{H}), 8.16-8.29$ (m, 4H), 8.38-8.42 (m, 2H), $8.98(\mathrm{~d}, J=8.4 \mathrm{~Hz}, 2 \mathrm{H}), 9.69(\mathrm{~s}, 1 \mathrm{H}) ;{ }^{13} \mathrm{C} \mathrm{NMR}\left(75 \mathrm{MHz}, \mathrm{CDCl}_{3}\right)$ : $\delta 124.1,124.3,125.2,127.2,127.3,129.2,129.3,131.8,133.8,136.2,137.1,144.5,150.5,162.3$, 172.0; HRMS (EI) Calcd. for $\mathrm{C}_{17} \mathrm{H}_{12} \mathrm{~N}_{2} \mathrm{O}_{4} \mathrm{~S}$ (M): 340.0517. Found: 340.0519 .
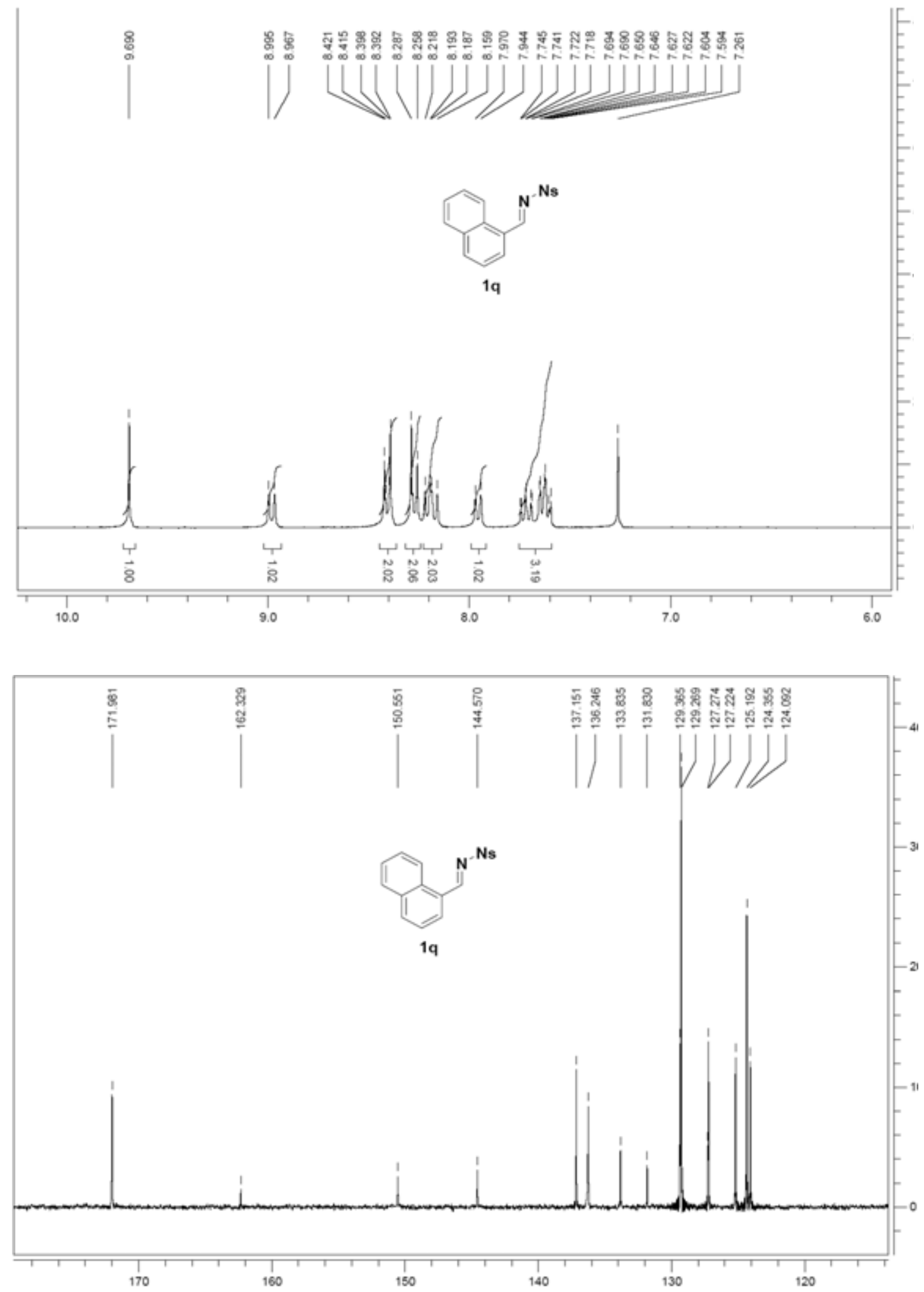
(B) General Procedure for the Catalytic Asymmetric Friedel-Crafts Reaction And Analytical Data of the Products

$N$-[Indol-3-yl-(4-nitrophenyl)methyl]-4-methylbenzenesulfonamide (2a): In a dried Schlenk tube, $\mathrm{Cu}(\mathrm{OTf})_{2}(98 \%, 14.8 \mathrm{mg}, 0.04 \mathrm{mmol})$, and $(S)$-Bn-bisoxazoline (21.7 $\left.\mathrm{mg}, 0.06 \mathrm{mmol}\right)$ were dissolved in $\mathrm{CH}_{2} \mathrm{Cl}_{2}(5 \mathrm{~mL})$ under $\mathrm{N}_{2}$ atmosphere. The solution was stirred at room temperature for $2 \mathrm{~h}$, then $4 \AA$ molecular sieves $(100 \mathrm{mg})$ was added and stirred for another 10 minutes. Subsequently, the $N$-(4-nitrobenzylidene)-4-methylbenzenesulfonamide (1a) (122 mg, $0.4 \mathrm{mmol})$ and indole (234 $\mathrm{mg}, 2.0 \mathrm{mmol}$ ) were added, and the reaction mixture was stirred at $20{ }^{\circ} \mathrm{C}$. After the reaction was completed (monitored by TLC), the solvent was removed under vacuum and the residue was purified by flash column chromatography on silica gel eluted with ethyl acetate/petroleum ether $(1: 3, \mathrm{v} / \mathrm{v})]$ to afford the product $N$-[indol-3-yl-(4-nitrophenyl)methyl]-4-methylbenzenesulfonamide (2a) as white powder, $158 \mathrm{mg}, 94 \%$ yield, mp: $211-213{ }^{\circ} \mathrm{C},[\alpha]^{20}{ }_{\mathrm{D}}=-22.7(\mathrm{c} 1.06$, AcOEt), 95\% ee [Daicel Chiralcel OD column $(25 \mathrm{~cm} \times 0.46 \mathrm{~cm} \mathrm{ID}), n$-hexane $/ \mathrm{i}-\mathrm{PrOH}=80: 20,1.0 \mathrm{~mL} / \mathrm{min}, 254$ $\mathrm{nm} ; t_{\mathrm{R}}=33.85 \mathrm{~min}$ and $\left.59.96 \mathrm{~min}\right] ;{ }^{1} \mathrm{H}$ NMR $\left(300 \mathrm{MHz}, \mathrm{CDCl}_{3}\right): \delta 2.41(\mathrm{~s}, 3 \mathrm{H}), 5.11(\mathrm{~d}, J=6.0 \mathrm{~Hz}$, $1 \mathrm{H}), 5.91(\mathrm{~d}, J=6.0 \mathrm{~Hz}, 1 \mathrm{H}), 6.59(\mathrm{~d}, J=2.4 \mathrm{~Hz}, 1 \mathrm{H}), 7.02(\mathrm{t}, J=7.5 \mathrm{~Hz}, 1 \mathrm{H}), 7.09$ (d, $J=7.8 \mathrm{~Hz}$, 1H), $7.21(\mathrm{t}, J=7.2 \mathrm{~Hz}, 3 \mathrm{H}), 7.34(\mathrm{~d}, J=8.1 \mathrm{~Hz}, 1 \mathrm{H}), 7.52(\mathrm{~d}, J=9.0 \mathrm{~Hz}, 2 \mathrm{H}), 7.62(\mathrm{~d}, J=9.0 \mathrm{~Hz}$, 2H), 8.09 (s, 2H), $8.11(\mathrm{~s}, 1 \mathrm{H}) ;{ }^{13} \mathrm{C}$ NMR (75 MHz, DMSO-d $): \delta 20.7,53.9,111.5,114.3,118.7$, $118.8,121.4,123.0,124.0,125.3,126.5,128.3,129.0,136.4,138.3,142.2,146.1,149.3$; HRMS (ESI) Calcd. for $\mathrm{C}_{22} \mathrm{H}_{19} \mathrm{~N}_{3} \mathrm{NaO}_{4} \mathrm{~S}(\mathrm{M}+\mathrm{Na})$ : 444.0988. Found: 444.0993. 


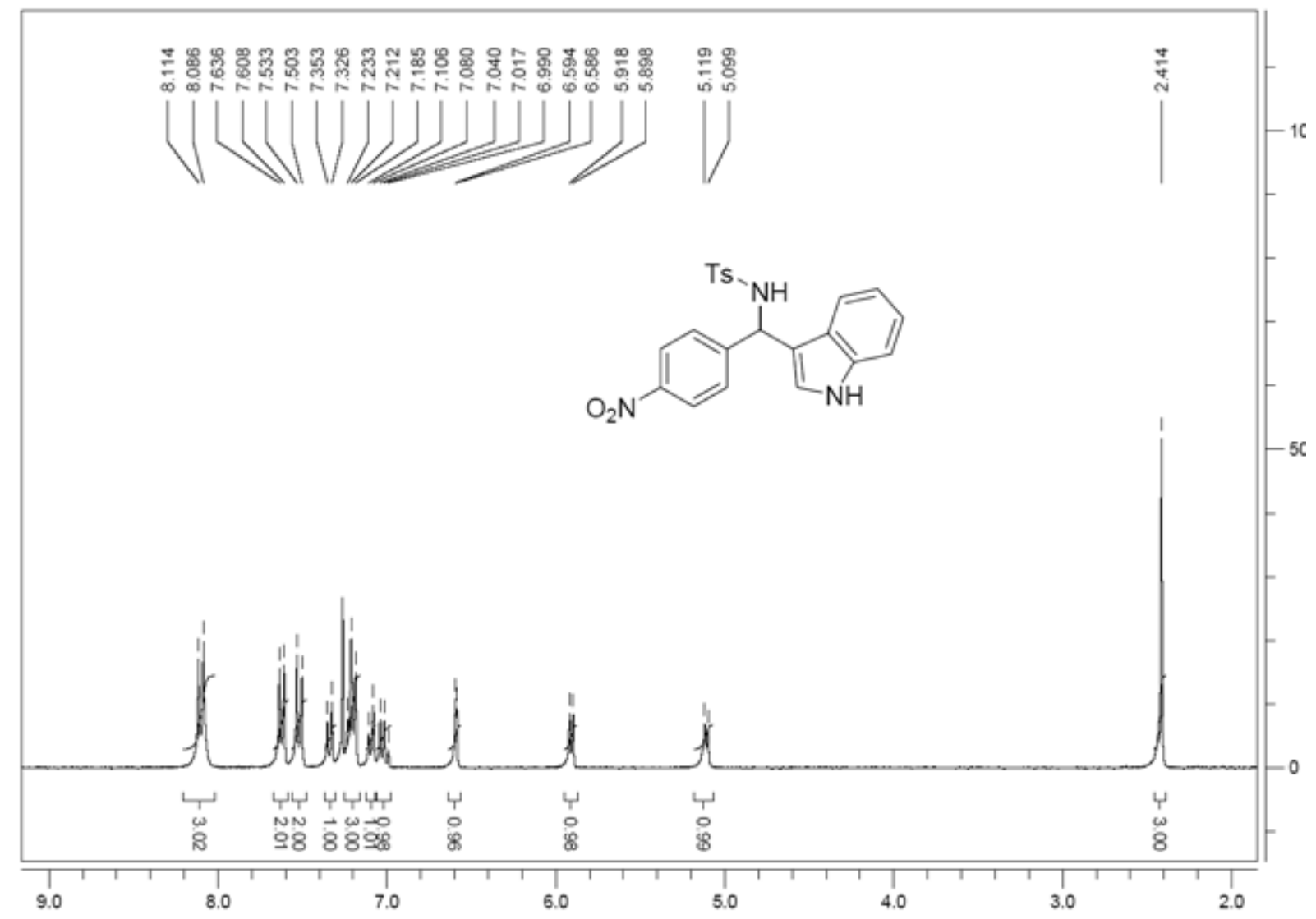

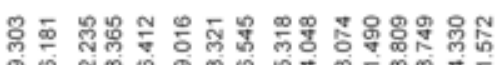

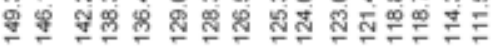

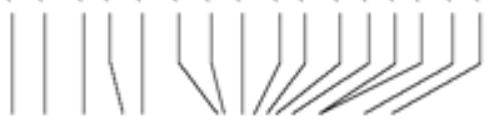

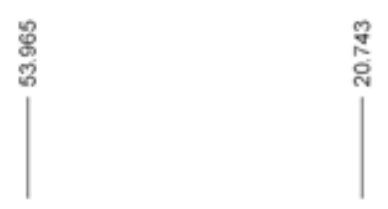<smiles>CNC(c1ccc([N+](=O)[O-])cc1)c1c[nH]c2ccccc12</smiles>

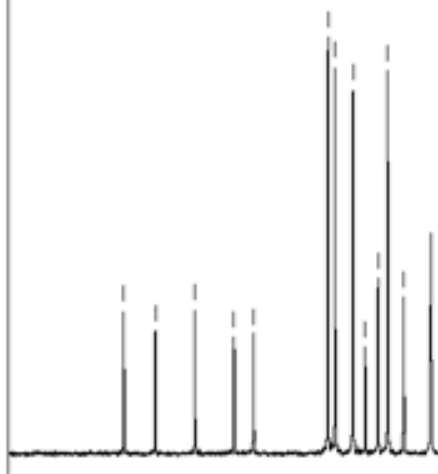

150

100 

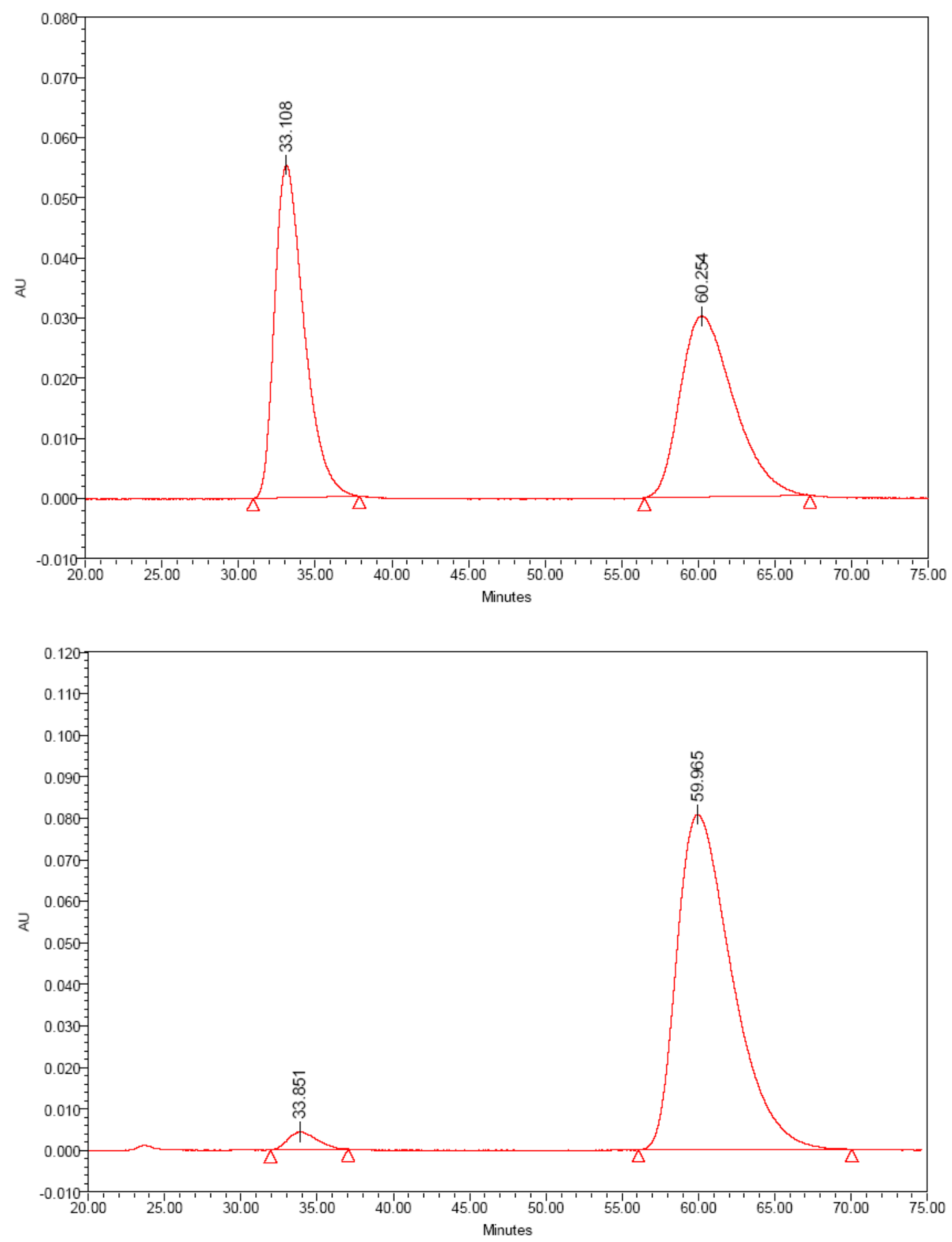

$N$-[Indol-3-yl-(3-nitrophenyl)methyl]-4-methylbenzenesulfonamide (2b): white powder, 94\% yield, mp: $202-204{ }^{\circ} \mathrm{C},[\alpha]^{20}{ }_{\mathrm{D}}=+10.4$ (c 1.8, AcOEt), 94\% ee [Daicel Chiralpak AD-H column $(25 \mathrm{~cm} \times 0.46 \mathrm{~cm} \mathrm{ID}), n$-hexane $/ \mathrm{i}-\mathrm{PrOH}=70: 30,1.0 \mathrm{~mL} / \mathrm{min}, 254 \mathrm{~nm} ; t_{\mathrm{R}}=27.93 \mathrm{~min}$ and 36.20 min]; ${ }^{1} \mathrm{H}$ NMR (300 MHz, $\left.\mathrm{CDCl}_{3}\right): \delta 2.38$ (s, 3H), 5.11 (d, J=5.4 Hz, 1H), $5.94(\mathrm{~d}, J=5.4 \mathrm{~Hz}, 1 \mathrm{H})$, $6.61(\mathrm{~d}, J=3.0 \mathrm{~Hz}, 1 \mathrm{H}), 7.03(\mathrm{t}, J=7.5 \mathrm{~Hz}, 1 \mathrm{H}), 7.15-7.24(\mathrm{~m}, 4 \mathrm{H}), 7.34(\mathrm{~d}, J=8.4 \mathrm{~Hz}, 1 \mathrm{H}), 7.44$ (t, $J=7.5 \mathrm{~Hz}, 1 \mathrm{H}), 7.60(\mathrm{~d}, J=8.4 \mathrm{~Hz}, 2 \mathrm{H}), 7.73$ (d, $J=7.5 \mathrm{~Hz}, 1 \mathrm{H}), 8.06(\mathrm{~d}, J=8.4 \mathrm{~Hz}, 2 \mathrm{H}), 8.08$ 
(s, 1H); ${ }^{13} \mathrm{C}$ NMR (75 MHz, DMSO-d $): \delta 20.7,53.8,111.5,114.6,118.7,118.8,121.4,121.7$, 124.0, 125.3, 126.4, 128.9, 129.3, 133.9, 136.4, 138.3, 142.0, 143.5, 147.5; HRMS (ESI) Calcd. for $\mathrm{C}_{22} \mathrm{H}_{23} \mathrm{~N}_{4} \mathrm{O}_{4} \mathrm{~S}\left(\mathrm{M}+\mathrm{NH}_{4}\right): 439.1435$. Found: 439.1435 .
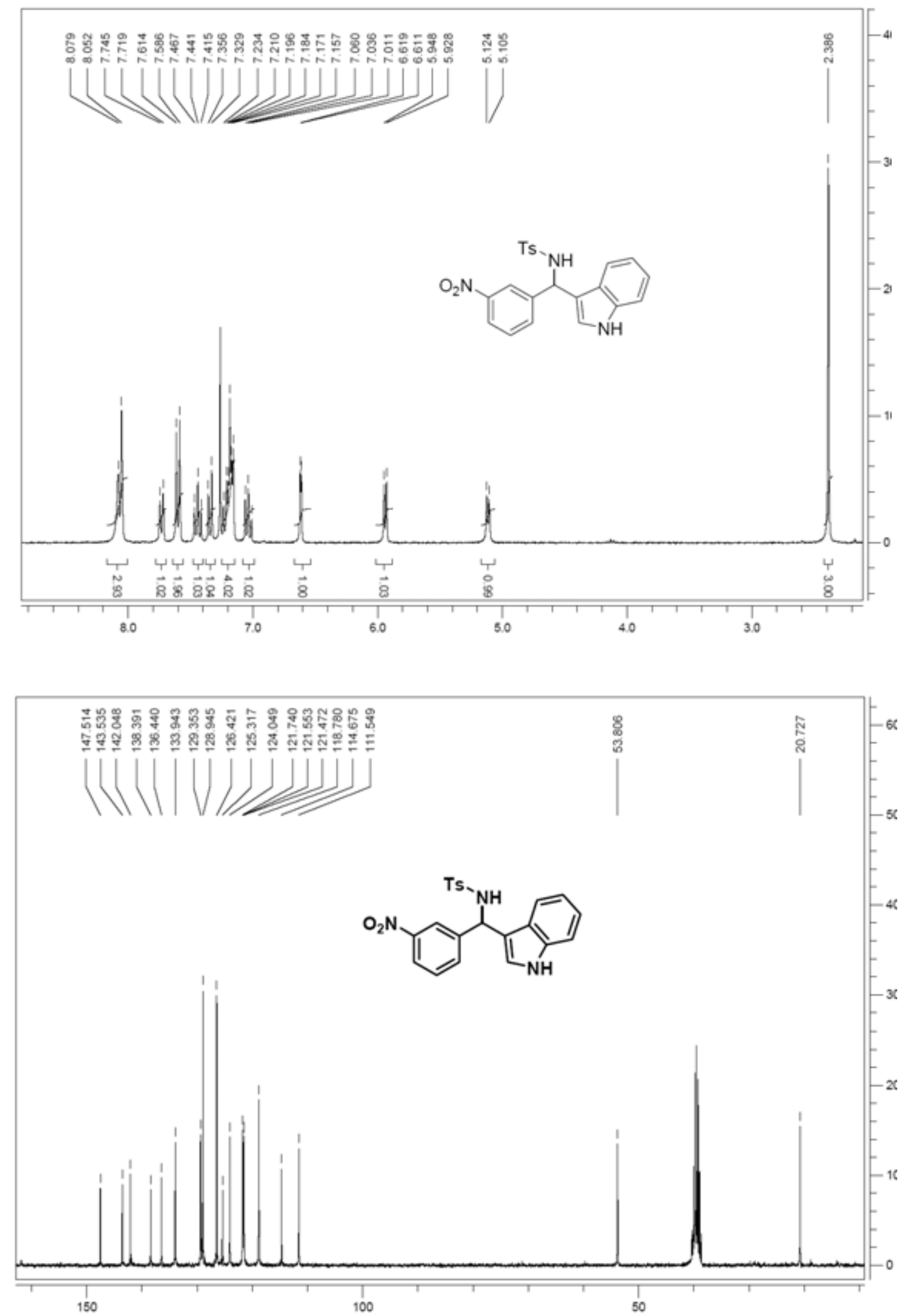

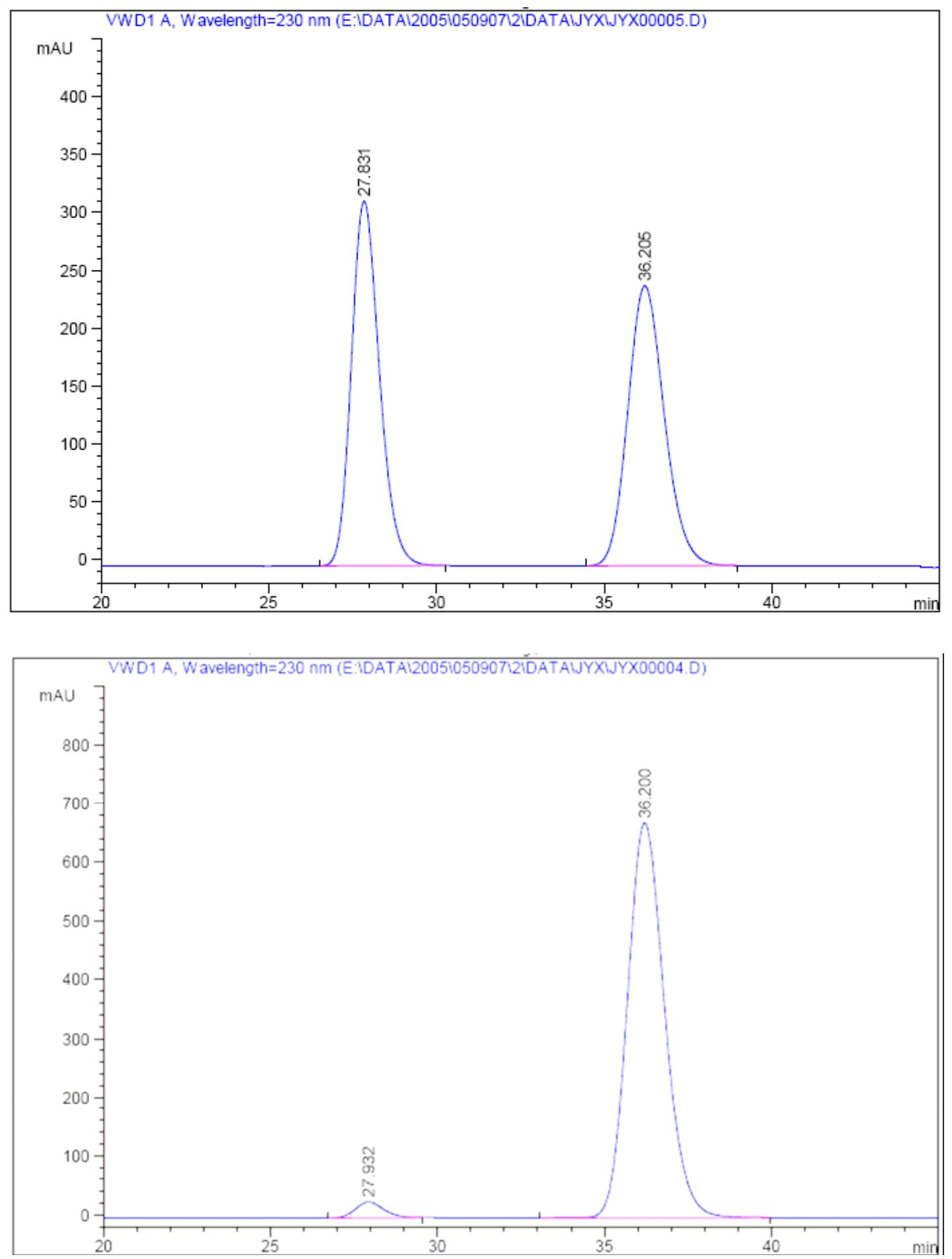

$N$-[Indol-3-yl-(4-chlorophenyl)methyl]-4-methylbenzenesulfonamide (2c): white powder, $75 \%$ yield, mp: $180-182{ }^{\circ} \mathrm{C},[\alpha]^{20}{ }_{\mathrm{D}}=-18.0\left(\mathrm{c} 1.1, \mathrm{CH}_{2} \mathrm{Cl}_{2}\right), 94 \%$ ee [Daicel Chiralcel OD column $(25 \mathrm{~cm} \times 0.46 \mathrm{~cm} \mathrm{ID}), n$-hexane $/ i-\mathrm{PrOH}=80: 20,1.0 \mathrm{~mL} / \mathrm{min}, 254 \mathrm{~nm} ; t_{\mathrm{R}}=20.13 \mathrm{~min}$ and 37.21 min]; ${ }^{1} \mathrm{H}$ NMR (300 MHz, DMSO-d $): \delta 2.29$ (s, 3H), $5.75(\mathrm{~d}, J=9.0 \mathrm{~Hz}, 1 \mathrm{H}), 6.78(\mathrm{~d}, J=1.8 \mathrm{~Hz}$, 1H), 6.91 (t, $J=7.5 \mathrm{~Hz}, 1 \mathrm{H}), 7.06$ (t, $J=7.2 \mathrm{~Hz}, 1 \mathrm{H}), 7.13$ (d, $J=8.1 \mathrm{~Hz}, 2 \mathrm{H}), 7.20$ (d, $J=8.4 \mathrm{~Hz}$, 2H), 7.26-7.35 (m, 4H), $7.50(\mathrm{~d}, J=8.4 \mathrm{~Hz}, 2 \mathrm{H}), 8.53(\mathrm{~d}, J=8.7 \mathrm{~Hz}, 1 \mathrm{H}), 10.94(\mathrm{~s}, 1 \mathrm{H}) ;{ }^{13} \mathrm{C} \mathrm{NMR}$ 
(75 MHz, DMSO-d $\left.{ }_{6}\right): \delta 20.8,53.8,111.4,115.1,118.6,118.8,121.2,123.7,125.3,126.4,127.7$, 128.9, 131.2, 136.3, 138.6, 140.5, 141.9; ESI MS m/z $409\left(\mathrm{M}^{-}-1\right)$; Anal. Calcd. for $\mathrm{C}_{22} \mathrm{H}_{19} \mathrm{ClN}_{2} \mathrm{O}_{2} \mathrm{~S}$ : C, 64.30; H, 4.66; N, 6.82. Found: C, 63.95; H, 4.57; N, 6.66.
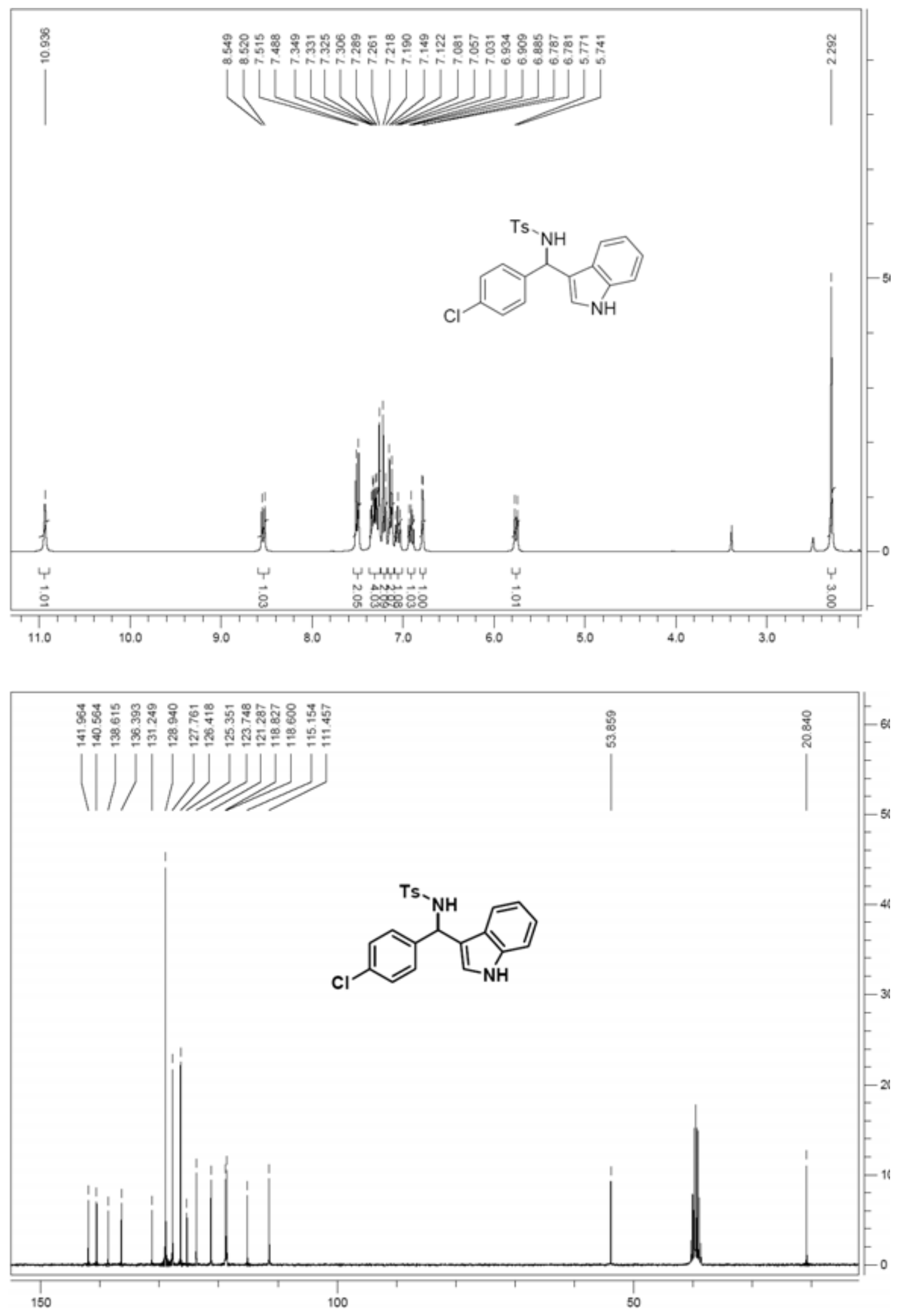

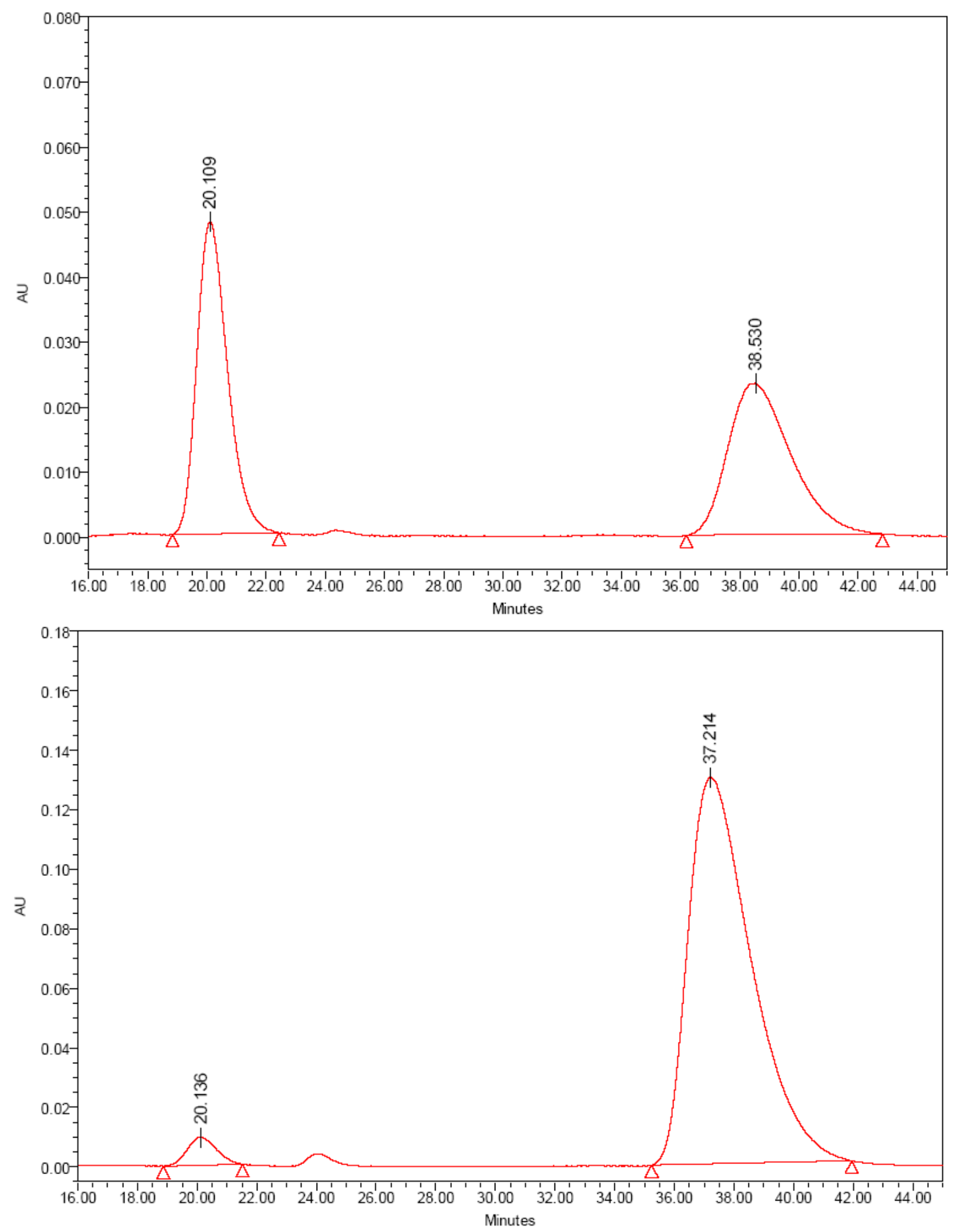

$N$-[Indol-3-yl-(3-chlorophenyl)methyl]-4-methylbenzenesulfonamide (2d): white powder, $79 \%$ yield, mp: $177-179{ }^{\circ} \mathrm{C},[\alpha]^{20}{ }_{\mathrm{D}}=-24.4\left(\mathrm{c} 0.8, \mathrm{CH}_{2} \mathrm{Cl}_{2}\right), 95 \%$ ee [Daicel Chiralcel OD column $(25 \mathrm{~cm} \times 0.46 \mathrm{~cm} \mathrm{ID}), n$-hexane $/ \mathrm{i}-\mathrm{PrOH}=80: 20,1.0 \mathrm{~mL} / \mathrm{min}, 254 \mathrm{~nm} ; t_{\mathrm{R}}=19.39 \mathrm{~min}$ (minor) and $23.69 \mathrm{~min}$ ]; ${ }^{1} \mathrm{H}$ NMR (300 MHz, $\left.\mathrm{CDCl}_{3}\right): \delta 2.39$ (s, 3H), $5.03(\mathrm{~d}, J=6.6 \mathrm{~Hz}, 1 \mathrm{H}), 5.83$ (d, $J=6.6$ Hz, 1H), $6.65(\mathrm{~s}, 1 \mathrm{H}), 7.03(\mathrm{t}, J=7.2 \mathrm{~Hz}, 1 \mathrm{H}), 7.13-7.33(\mathrm{~m}, 9 \mathrm{H}), 7.57(\mathrm{~d}, J=8.1 \mathrm{~Hz}, 2 \mathrm{H}), 8.02(\mathrm{~s}$, $1 \mathrm{H}) ;{ }^{13} \mathrm{C}$ NMR (75 MHz, DMSO-d $\left.{ }_{6}\right): \delta 20.8,53.8,111.5,114.9,118.7,121.3,123.5,123.7,125.2$, 
125.7, 126.3, 126.5, 126.8, 128.9, 129.7, 132.7, 136.3, 138.3, 142.1, 143.8; ESI MS m/z $409\left(\mathrm{M}^{-}-1\right)$;

Anal. Calcd. for $\mathrm{C}_{22} \mathrm{H}_{19} \mathrm{ClN}_{2} \mathrm{O}_{2} \mathrm{~S}: \mathrm{C}, 64.30 ; \mathrm{H}, 4.66 ; \mathrm{N}, 6.82$. Found: C, 64.07; H, 4.81; N, 6.71 .
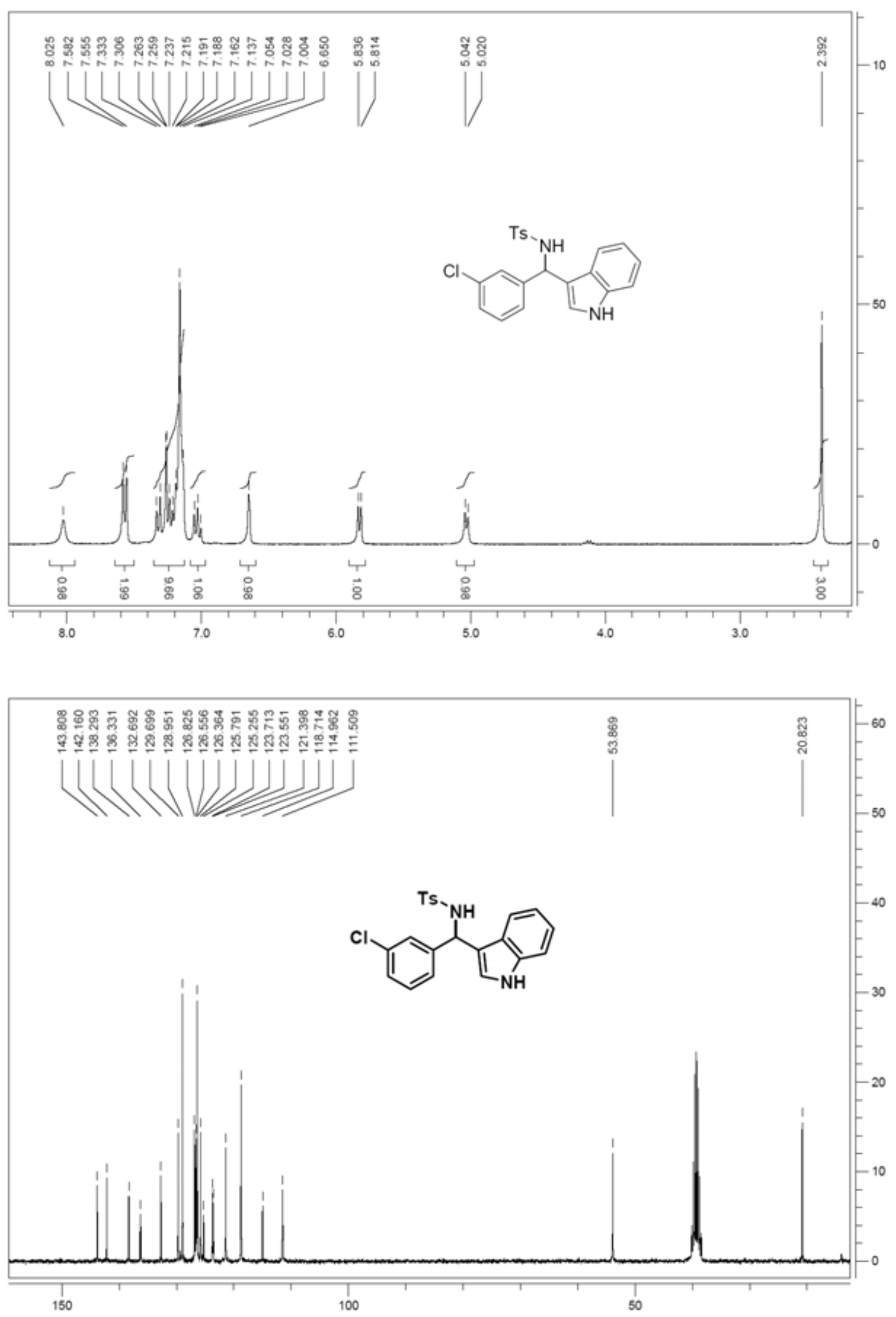

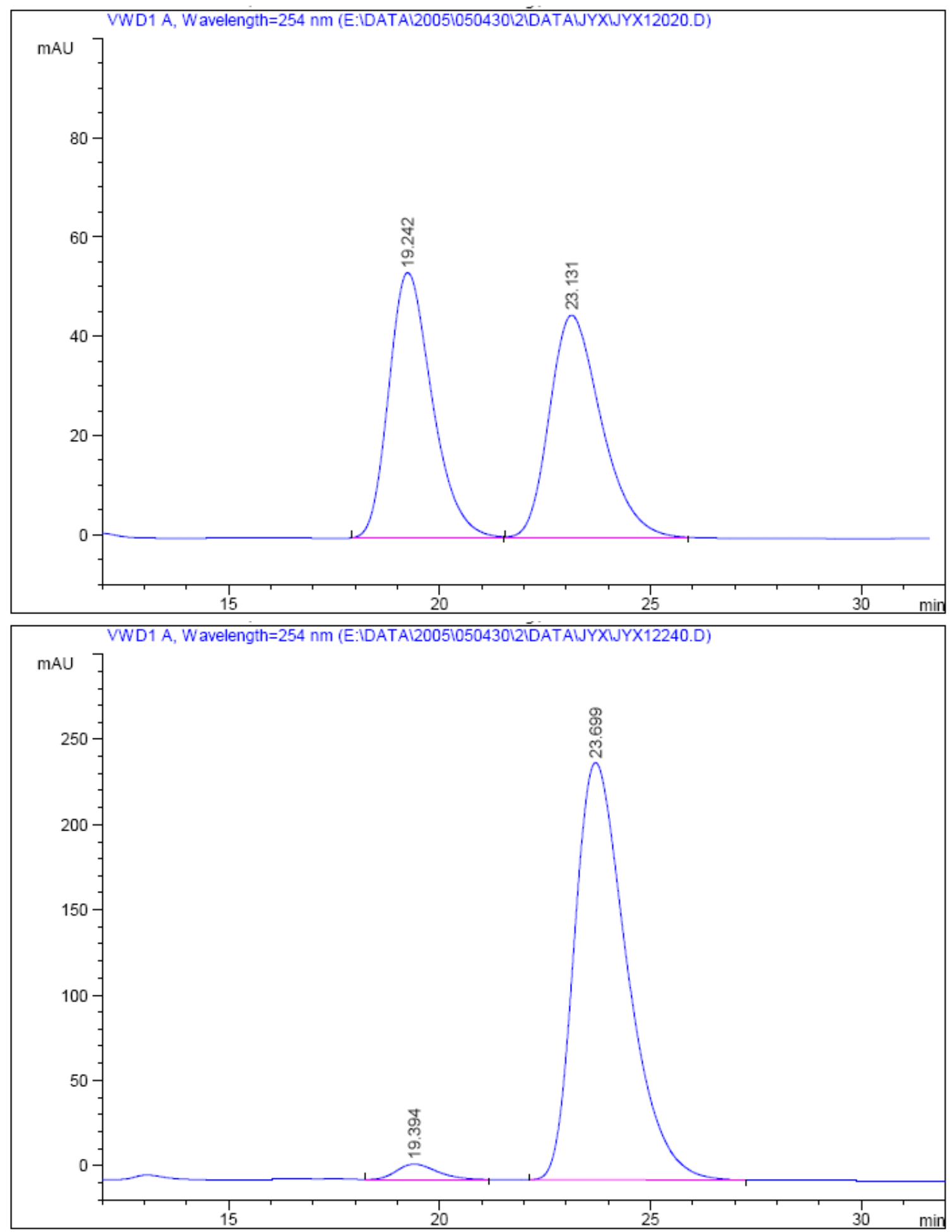

$N$-[Indol-3-yl-(2-chlorophenyl)methyl]-4-methylbenzenesulfonamide (2e): white powder, $72 \%$ yield, mp: $177-179{ }^{\circ} \mathrm{C},[\alpha]^{20}{ }_{\mathrm{D}}=-54.3\left(\mathrm{c} 1.15, \mathrm{CH}_{2} \mathrm{Cl}_{2}\right), 94 \%$ ee [Daicel Chiralcel OD column $(25 \mathrm{~cm} \times 0.46 \mathrm{~cm} \mathrm{ID}), n$-hexane $/ \mathrm{i}-\mathrm{PrOH}=70: 30,1.0 \mathrm{~mL} / \mathrm{min}, 254 \mathrm{~nm} ; t_{\mathrm{R}}=12.58 \mathrm{~min}$ and 32.69 min]; ${ }^{1} \mathrm{H}$ NMR $\left(300 \mathrm{MHz}, \mathrm{CDCl}_{3}\right): \delta 2.39(\mathrm{~s}, 3 \mathrm{H}), 5.16(\mathrm{~d}, J=5.7 \mathrm{~Hz}, 1 \mathrm{H}), 6.21(\mathrm{~d}, J=5.7 \mathrm{~Hz}, 1 \mathrm{H})$, $6.98(\mathrm{~s}, 1 \mathrm{H}), 7.01(\mathrm{t}, J=7.5 \mathrm{~Hz}, 1 \mathrm{H}), 7.19-7.32(\mathrm{~m}, 8 \mathrm{H}), 7.49-7.52(\mathrm{~m}, 1 \mathrm{H}), 7.66(\mathrm{~d}, J=7.8 \mathrm{~Hz}$, 
2H), $8.02(\mathrm{~s}, 1 \mathrm{H}) ;{ }^{13} \mathrm{C}$ NMR $\left(75 \mathrm{MHz}, \mathrm{DMSO}-d_{6}\right): \delta 20.8,53.6,111.5,114.1,118.5,118.7,121.4$, 124.1, 125.6, 126.3, 126.9, 128.2, 128.8, 129.0, 129.2, 131.4, 136.3, 138.3, 138.8, 142.1; ESI MS m/z $409\left(\mathrm{M}^{-}-1\right)$; Anal. Calcd. for $\mathrm{C}_{22} \mathrm{H}_{19} \mathrm{ClN}_{2} \mathrm{O}_{2} \mathrm{~S}$ : C, 64.30; H, 4.66; N, 6.82. Found: $\mathrm{C}, 64.05 ; \mathrm{H}$, $4.87 ; \mathrm{N}, 6.65$.
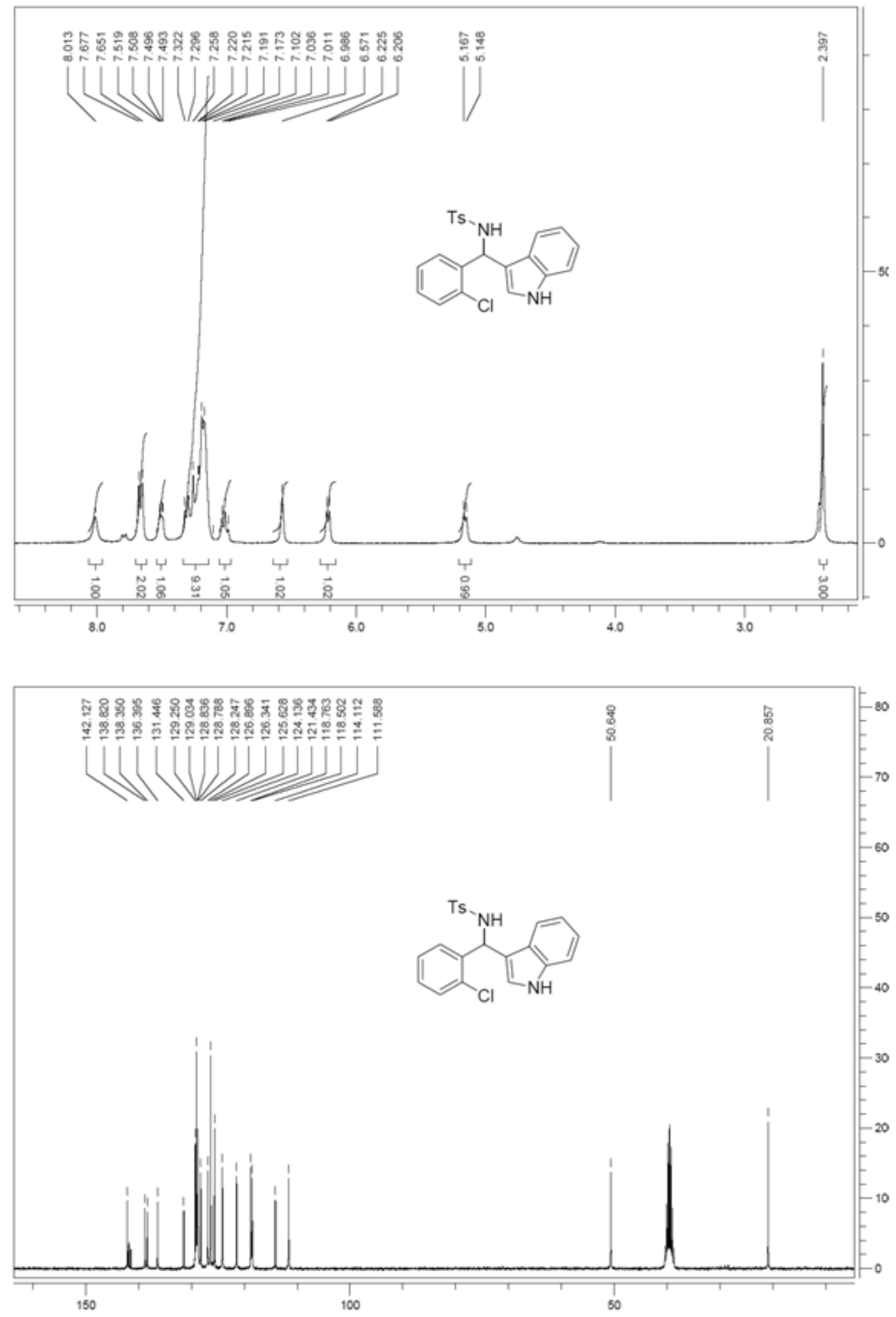

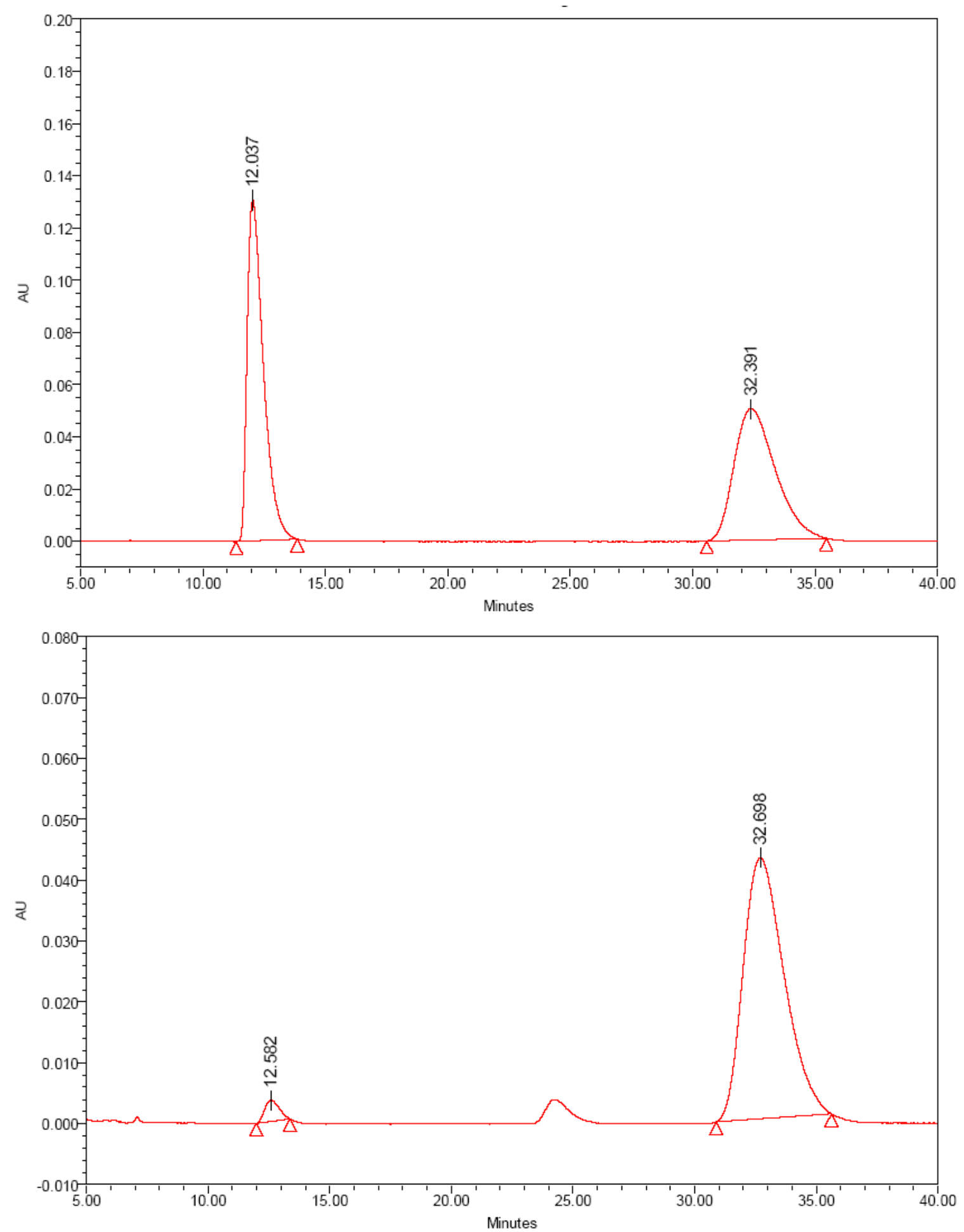

$N$-[Indol-3-yl-(4-bromophenyl)methyl]-4-methylbenzenesulfonamide (2f): white powder, 89\% yield, mp: $188-190{ }^{\circ} \mathrm{C},[\alpha]^{20}{ }_{\mathrm{D}}=-15.8$ ( $\mathrm{c} 1.35, \mathrm{CH}_{2} \mathrm{Cl}_{2}$ ), 96\% ee [Daicel Chiralcel OD column $(25 \mathrm{~cm} \times 0.46 \mathrm{~cm} \mathrm{ID}), n$-hexane $/ \mathrm{i}-\mathrm{PrOH}=76: 24,1.0 \mathrm{~mL} / \mathrm{min}, 254 \mathrm{~nm} ; t_{\mathrm{R}}=16.77 \mathrm{~min}$ and 28.17 min]; ${ }^{1} \mathrm{H}$ NMR $\left(300 \mathrm{MHz}, \mathrm{CDCl}_{3}\right): \delta 2.40(\mathrm{~s}, 3 \mathrm{H}), 5.03(\mathrm{~d}, J=6.6 \mathrm{~Hz}, 1 \mathrm{H}), 5.80(\mathrm{~d}, J=6.6 \mathrm{~Hz}, 1 \mathrm{H})$, $6.63(\mathrm{~d}, J=2.4 \mathrm{~Hz}, 1 \mathrm{H}), 7.01(\mathrm{t}, J=7.5 \mathrm{~Hz}, 1 \mathrm{H}), 7.11-7.22(\mathrm{~m}, 6 \mathrm{H}), 7.30-7.34(\mathrm{~m}, 3 \mathrm{H}), 7.55(\mathrm{~d}, J=$ 
$8.1 \mathrm{~Hz}, 2 \mathrm{H}), 8.0(\mathrm{~s}, 1 \mathrm{H}) ;{ }^{13} \mathrm{C}$ NMR $\left(75 \mathrm{MHz}, \mathrm{DMSO}-d_{6}\right): \delta 20.8,53.9,111.4,115.0,118.6,118.8$, 119.7, 121.3, 123.7, 125.3, 125.6, 126.4, 128.9, 129.3, 130.6, 136.3, 138.5, 140.9, 141.9; ESI MS m/z $501\left(\mathrm{M}^{-}+\mathrm{HCOOH}\right)$; Anal. Calcd. for $\mathrm{C}_{22} \mathrm{H}_{19} \mathrm{BrN}_{2} \mathrm{O}_{2} \mathrm{~S}: \mathrm{C}, 58.03 ; \mathrm{H}, 4.21 ; \mathrm{N}, 6.15$. Found: $\mathrm{C}$, $57.87 ; \mathrm{H}, 4.50 ; \mathrm{N}, 5.95$.
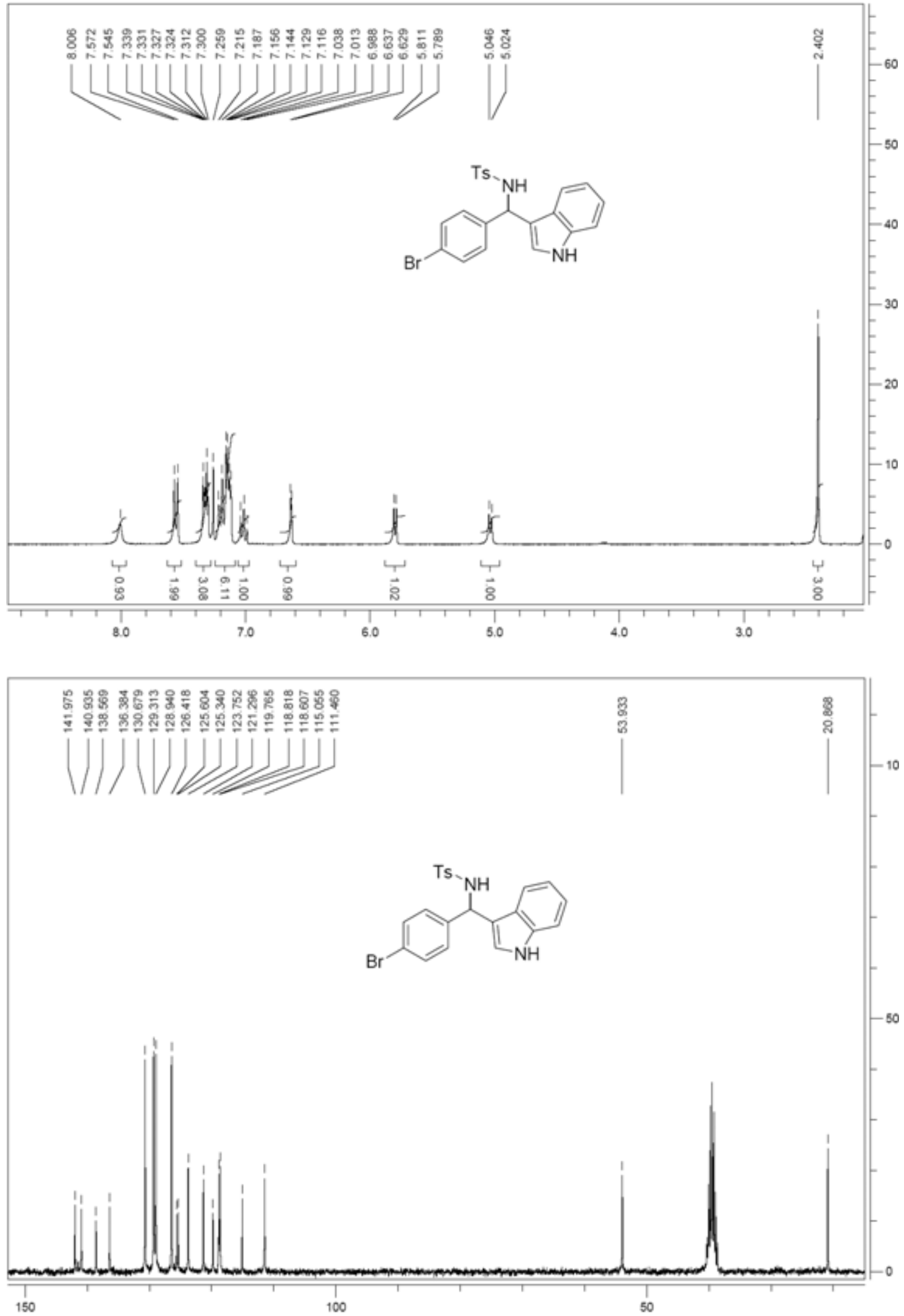

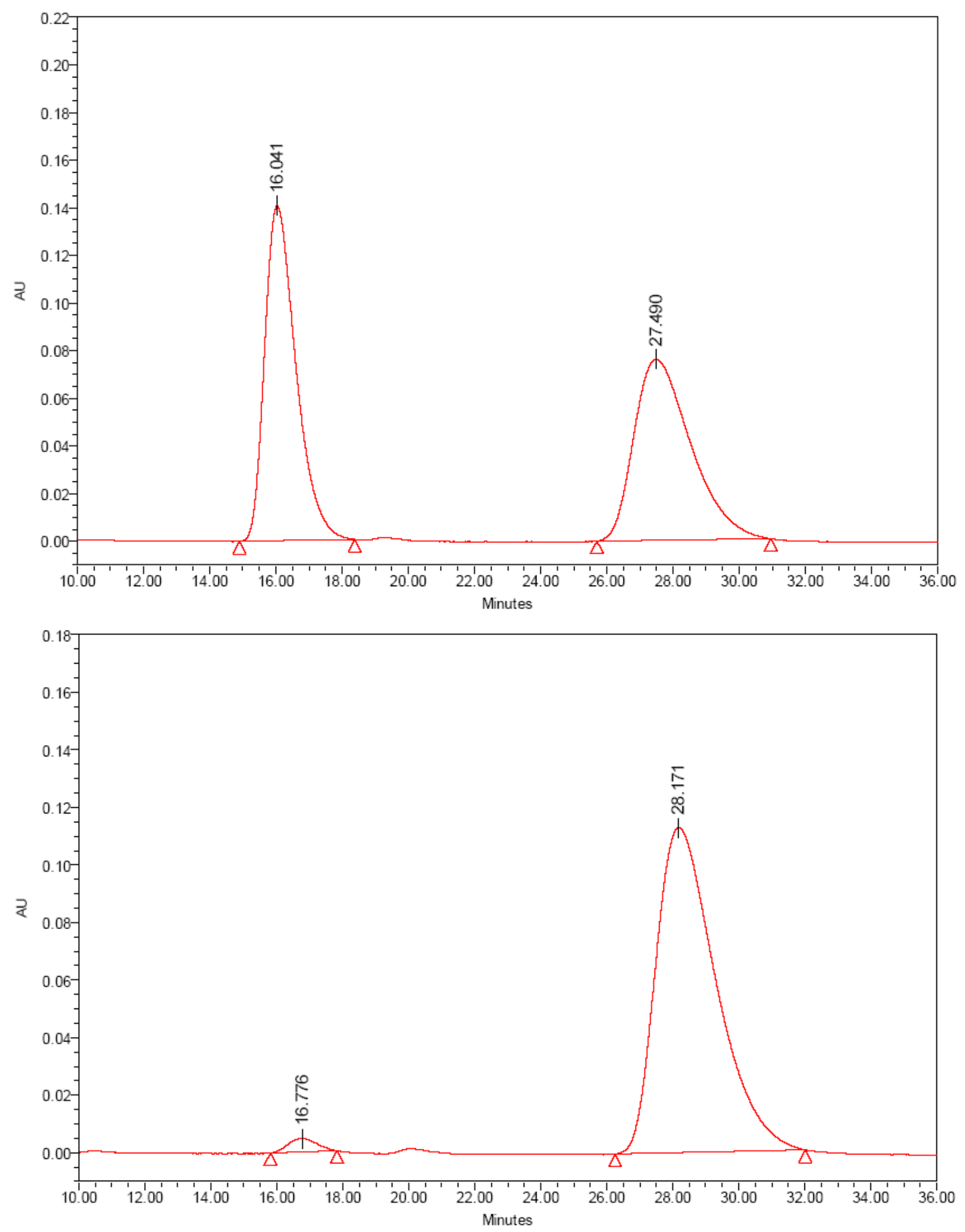

$N$-[Indol-3-yl-(3-bromophenyl)methyl]-4-methylbenzenesulfonamide (2g): white powder, 87\% yield, mp: $168-169{ }^{\circ} \mathrm{C},[\alpha]^{20}{ }_{\mathrm{D}}=-20.6\left(\mathrm{c} 1.6, \mathrm{CH}_{2} \mathrm{Cl}_{2}\right), 94 \%$ ee [Daicel Chiralpak AD-H column $(25 \mathrm{~cm} \times 0.46 \mathrm{~cm} \mathrm{ID}), n$-hexane $/ \mathrm{i}-\mathrm{PrOH}=70: 30,1.0 \mathrm{~mL} / \mathrm{min}, 254 \mathrm{~nm} ; t_{\mathrm{R}}=23.95 \mathrm{~min}$ and $27.62 \mathrm{~min}] ;{ }^{1} \mathrm{H}$ NMR $\left(300 \mathrm{MHz}, \mathrm{CDCl}_{3}\right): \delta 2.40(\mathrm{~s}, 3 \mathrm{H}), 5.01(\mathrm{~d}, J=6.3 \mathrm{~Hz}, 1 \mathrm{H}), 5.82(\mathrm{~d}, J=6.3$ $\mathrm{Hz}, 1 \mathrm{H}), 6.64(\mathrm{~d}, J=2.4 \mathrm{~Hz}, 1 \mathrm{H}), 7.01-7.22(\mathrm{~m}, 6 \mathrm{H}), 7.26-7.34(\mathrm{~m}, 4 \mathrm{H}), 7.56(\mathrm{~d}, J=8.1 \mathrm{~Hz}, 2 \mathrm{H})$, $8.02(\mathrm{~s}, 1 \mathrm{H}) ;{ }^{13} \mathrm{C}$ NMR $\left(75 \mathrm{MHz}, \mathrm{DMSO}-d_{6}\right): \delta 20.8,53.8,111.5,114.9,118.7,121.35,121.39$, 
123.7, 125.2, 126.1, 126.3, 128.9, 129.4, 129.7, 129.9, 136.3, 138.3, 142.1, 143.9; ESI MS m/z 501 $\left(\mathrm{M}^{-}+\mathrm{HCOOH}\right)$; Anal. Calcd. for $\mathrm{C}_{22} \mathrm{H}_{19} \mathrm{BrN}_{2} \mathrm{O}_{2} \mathrm{~S}: \mathrm{C}, 58.03 ; \mathrm{H}, 4.21 ; \mathrm{N}, 6.15$. Found: C, 57.85; $\mathrm{H}$, 4.01; N, 5.91 .
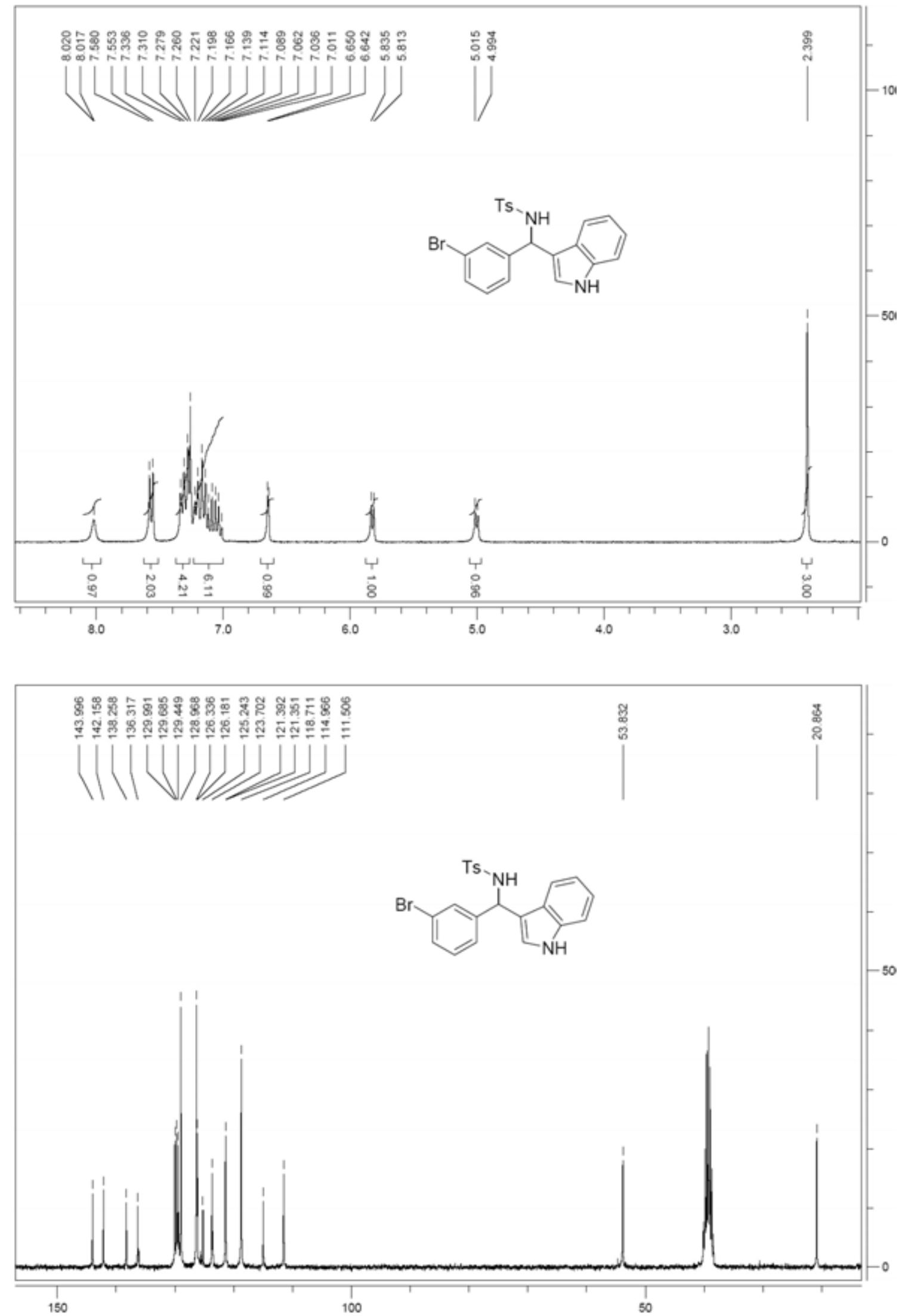

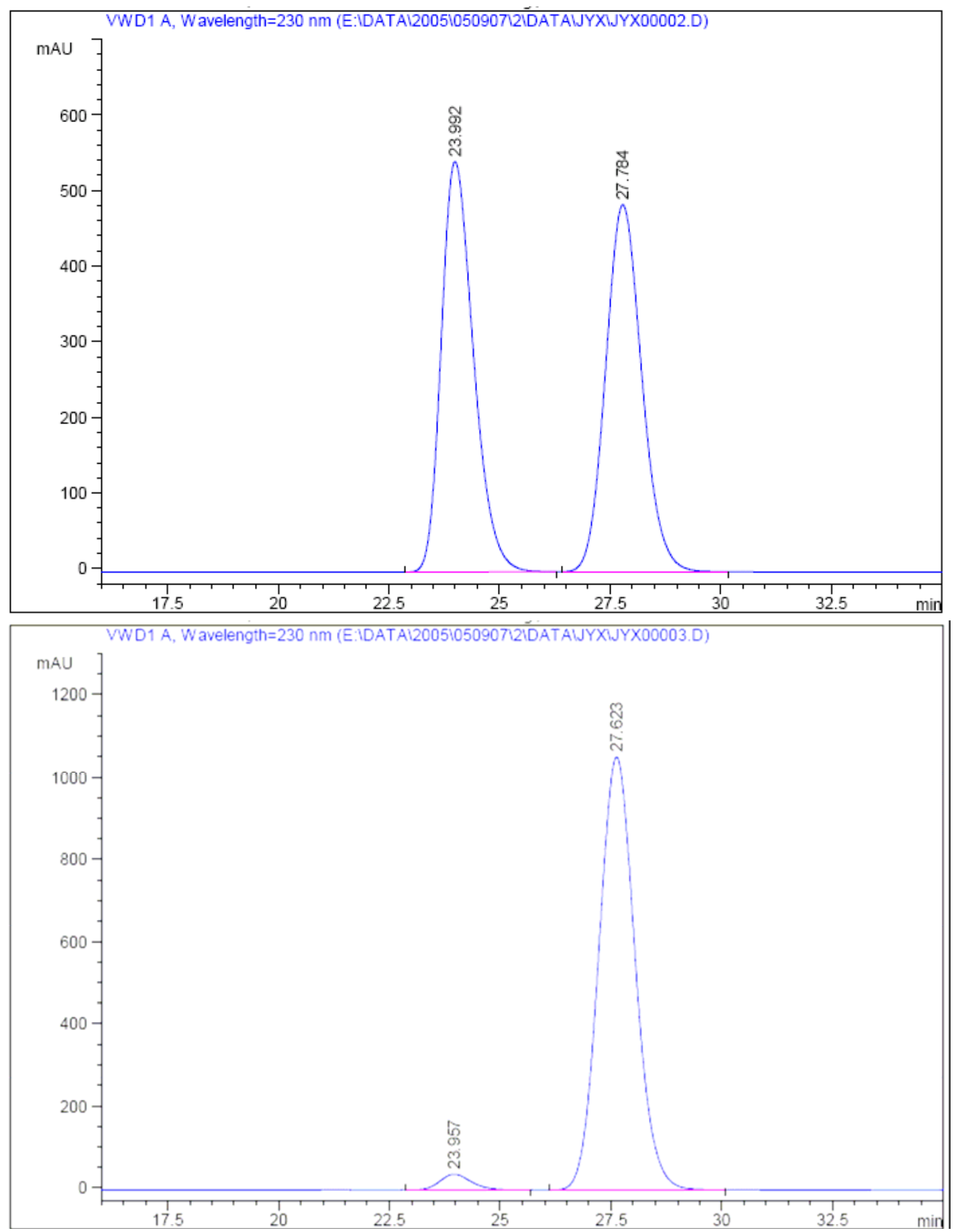

$N$-[Indol-3-yl-(2-bromophenyl)methyl]-4-methylbenzenesulfonamide (2h): white powder, 78\% yield, mp: $199-201^{\circ} \mathrm{C},[\alpha]^{20}{ }_{\mathrm{D}}=-74.3\left(\mathrm{c} 1.9, \mathrm{CH}_{2} \mathrm{Cl}_{2}\right), 94 \%$ ee [Daicel Chiralcel OD column column $(25 \mathrm{~cm} \times 0.46 \mathrm{~cm} \mathrm{ID}), n$-hexane $/ \mathrm{i}-\mathrm{PrOH}=80: 20,1.0 \mathrm{~mL} / \mathrm{min}, 254 \mathrm{~nm} ; t_{\mathrm{R}}=12.66 \mathrm{~min}$ and $33.95 \mathrm{~min}$ ]; ${ }^{1} \mathrm{H}$ NMR (300 MHz, $\left.\mathrm{CDCl}_{3}\right): \delta 2.40$ (s, 3H), $5.12(\mathrm{~d}, J=6.0 \mathrm{~Hz}, 1 \mathrm{H}), 6.19$ (d, $J=6.0$ $\mathrm{Hz}, 1 \mathrm{H}), 6.53(\mathrm{~d}, J=1.8 \mathrm{~Hz}, 1 \mathrm{H}), 6.98-7.11(\mathrm{~m}, 2 \mathrm{H}), 7.15-7.25(\mathrm{~m}, 5 \mathrm{H}), 7.31(\mathrm{~d}, J=8.1 \mathrm{~Hz}, 1 \mathrm{H})$, $7.44(\mathrm{dd}, J=1.2,7.8 \mathrm{~Hz}, 1 \mathrm{H}), 7.53(\mathrm{dd}, J=1.2,7.8 \mathrm{~Hz}, 1 \mathrm{H}), 7.69$ (d, $J=8.1 \mathrm{~Hz}, 2 \mathrm{H}), 8.0(\mathrm{~s}, 1 \mathrm{H})$; 
${ }^{13} \mathrm{C}$ NMR (75 MHz, DMSO-d $\left.{ }_{6}\right): \delta 20.8,53.1,111.5,114.0,118.5,118.8,121.5,122.7,124.2,125.6$, $126.3,127.4,128.5,128.9,129.0,132.0,136.3,138.1,140.2,142.2$; HRMS (EI) Calcd. for $\mathrm{C}_{22} \mathrm{H}_{19} \mathrm{BrN}_{2} \mathrm{O}_{2} \mathrm{~S}:$ 454.0345. Found: 454.0352 .
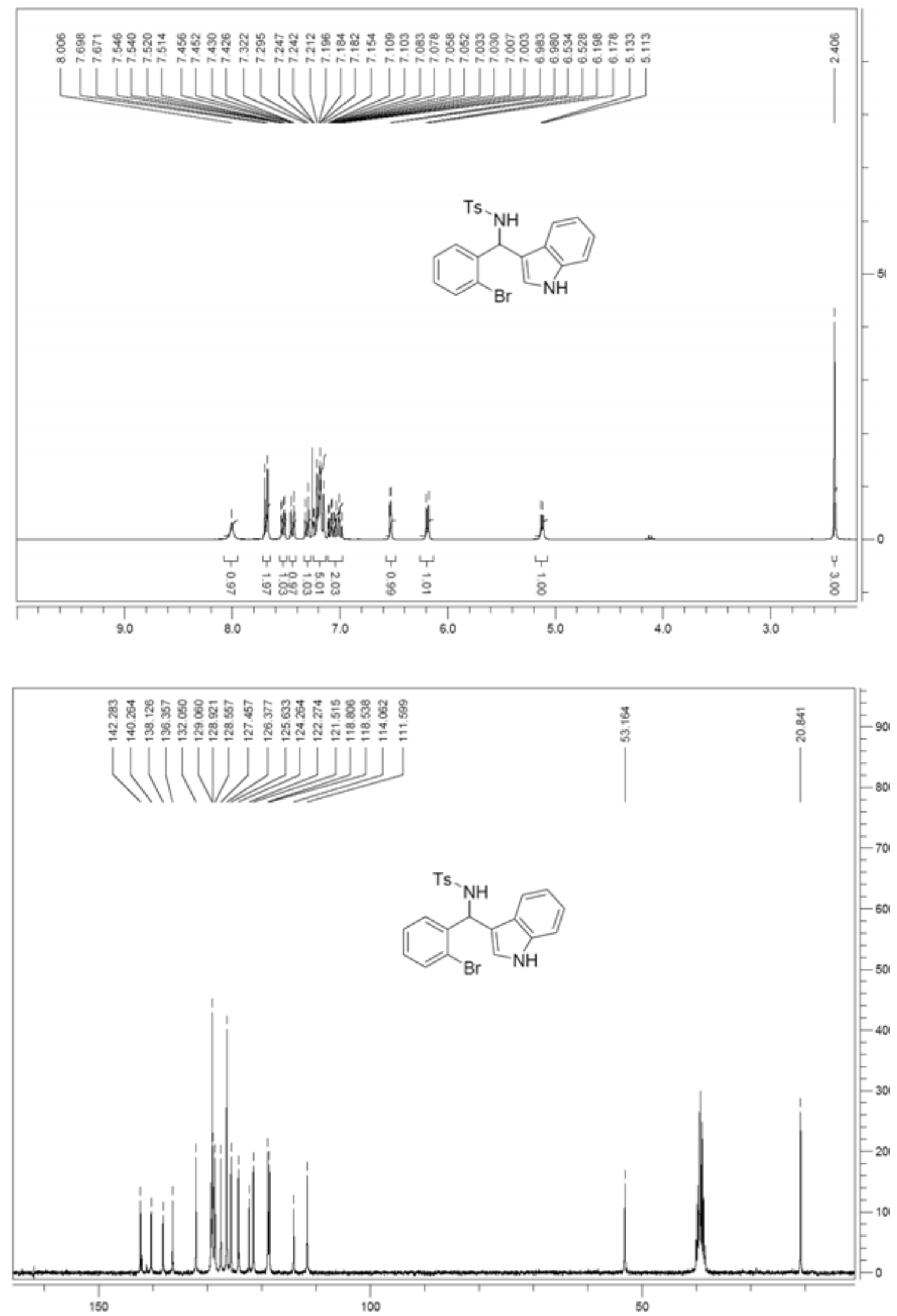

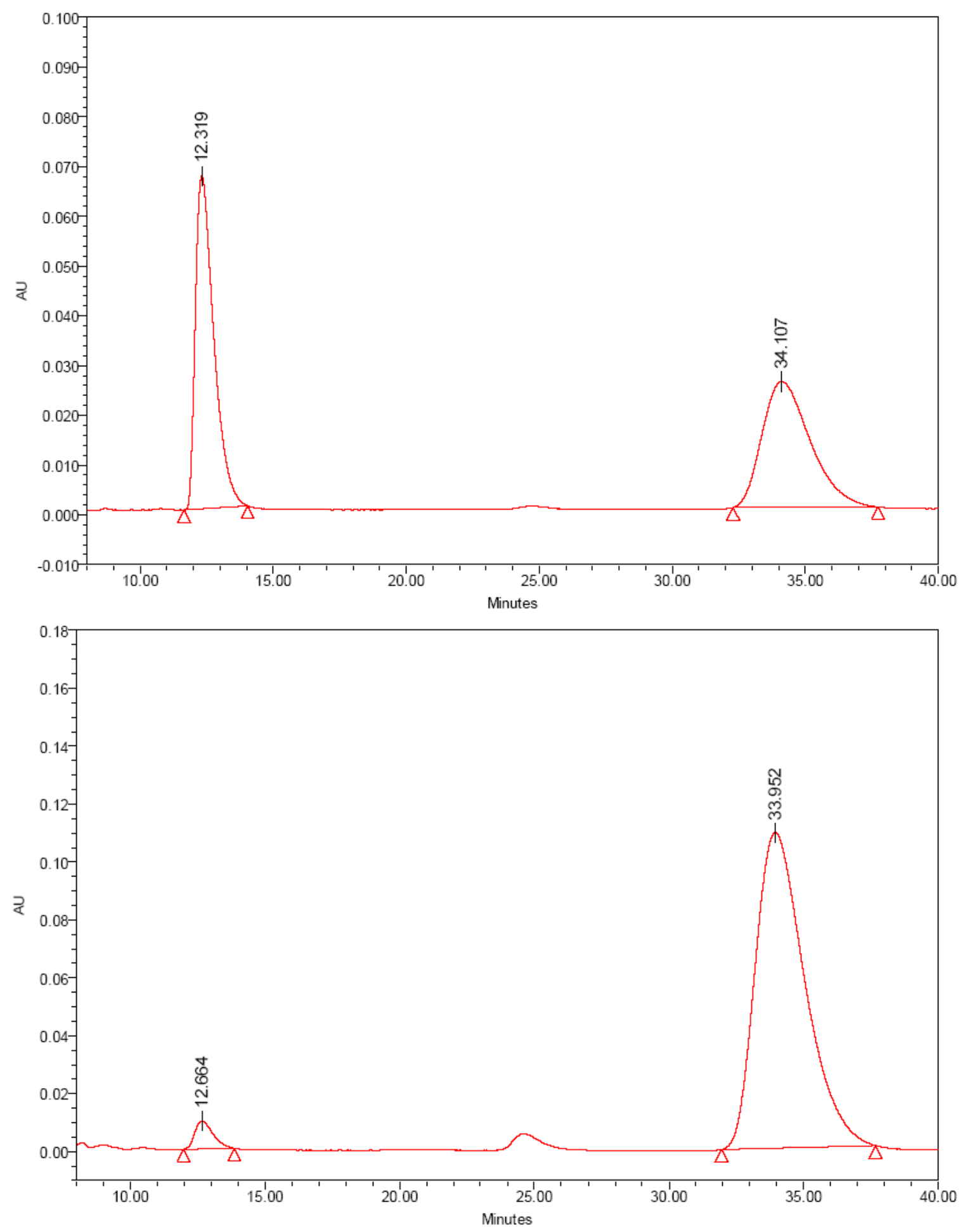

$N$-[Indol-3-yl-(3,4-dichlorophenyl)methyl]-4-methylbenzenesulfonamide (2i): white powder, $63 \%$ yield, mp: $207-209{ }^{\circ} \mathrm{C},[\alpha]^{20}{ }_{\mathrm{D}}=-8.4$ (c 1.3, AcOEt), 95\% ee [Daicel Chiralpak AD-H column $(25 \mathrm{~cm} \times 0.46 \mathrm{~cm} \mathrm{ID}), n$-hexane $/ \mathrm{i}-\mathrm{PrOH}=80: 20,1.0 \mathrm{~mL} / \mathrm{min}, 254 \mathrm{~nm} ; t_{\mathrm{R}}=20.65 \mathrm{~min}$ (minor) and $38.81 \mathrm{~min}] ;{ }^{1} \mathrm{H}$ NMR (300 MHz, $\left.\mathrm{CDCl}_{3}\right): \delta 2.40$ (s, 3H), $5.10(\mathrm{~d}, J=6.3 \mathrm{~Hz}, 1 \mathrm{H}), 5.78(\mathrm{~d}, J=6.3$ $\mathrm{Hz}, 1 \mathrm{H}), 6.62$ (d, $J=2.1 \mathrm{~Hz}, 1 \mathrm{H}), 7.03$ (t, $J=7.2 \mathrm{~Hz}, 1 \mathrm{H}), 7.10-7.33(\mathrm{~m}, 8 \mathrm{H}), 7.56$ (d, $J=7.5 \mathrm{~Hz}$, 2H), $8.04(\mathrm{~s}, 1 \mathrm{H}) ;{ }^{13} \mathrm{C}$ NMR (75 MHz, DMSO-d $): \delta 20.8,53.5,111.5,114.5,118.7,121.5,123.8$, 
125.2, 125.6, 126.4, 127.5, 128.9, 129.0, 129.2, 129.9, 130.6, 136.3, 138.1, 142.0, 142.9; HRMS (ESI) Calcd. for $\mathrm{C}_{22} \mathrm{H}_{18} \mathrm{Cl}_{2} \mathrm{~N}_{2} \mathrm{NaO}_{2} \mathrm{~S}(\mathrm{M}+\mathrm{Na}$ ): 467.0358. Found: 467.0355.

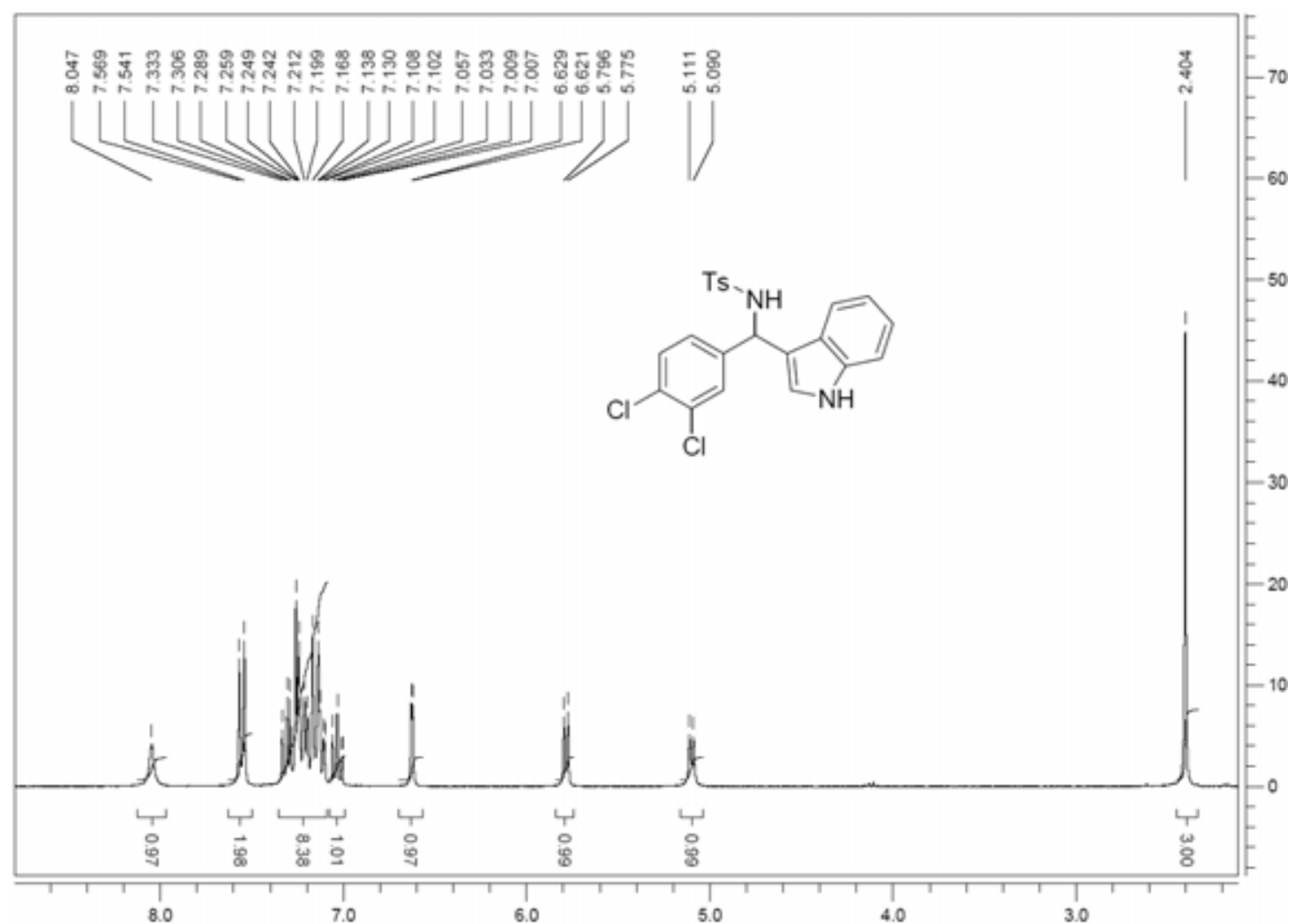

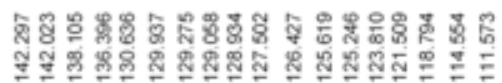
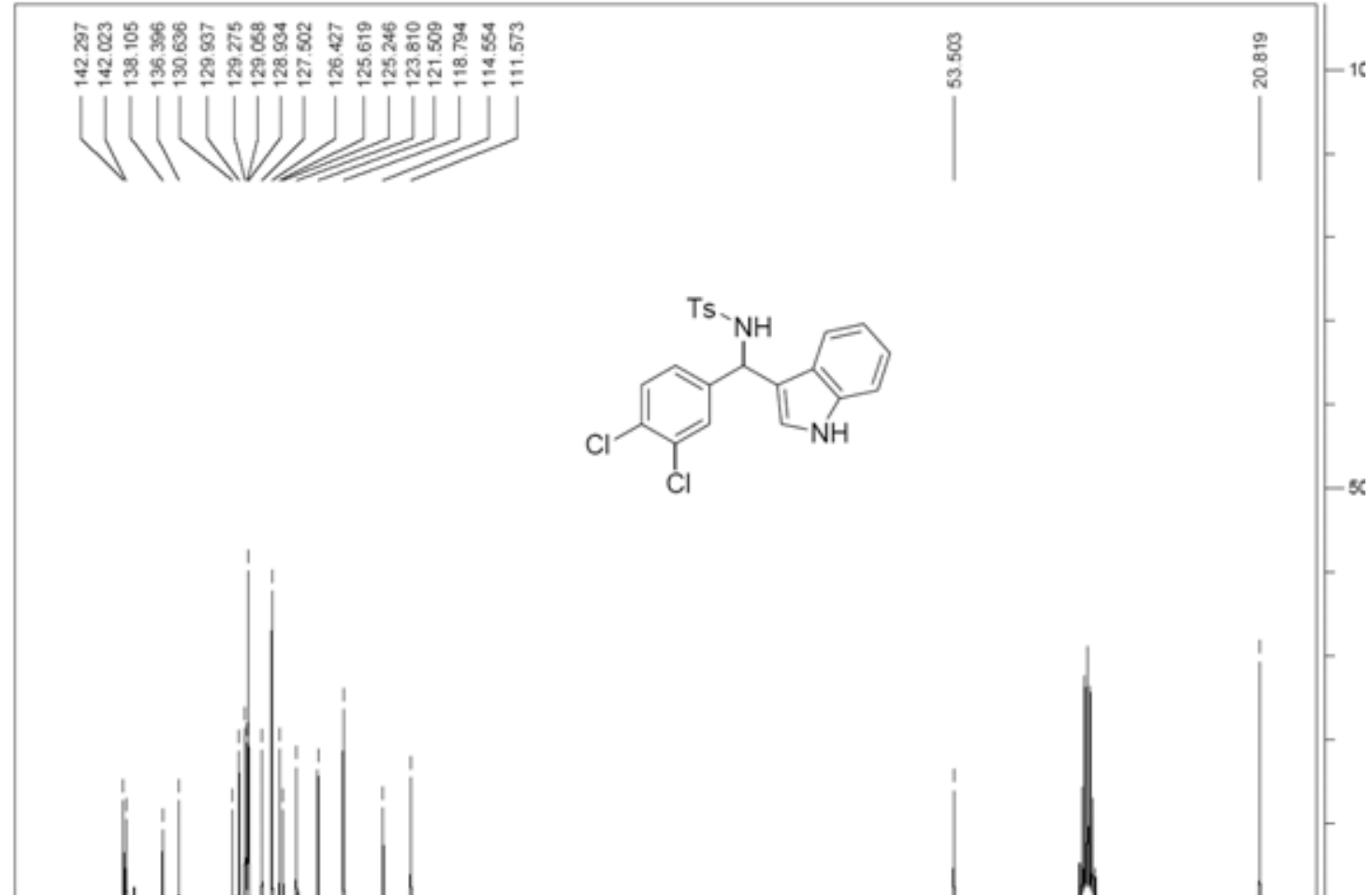

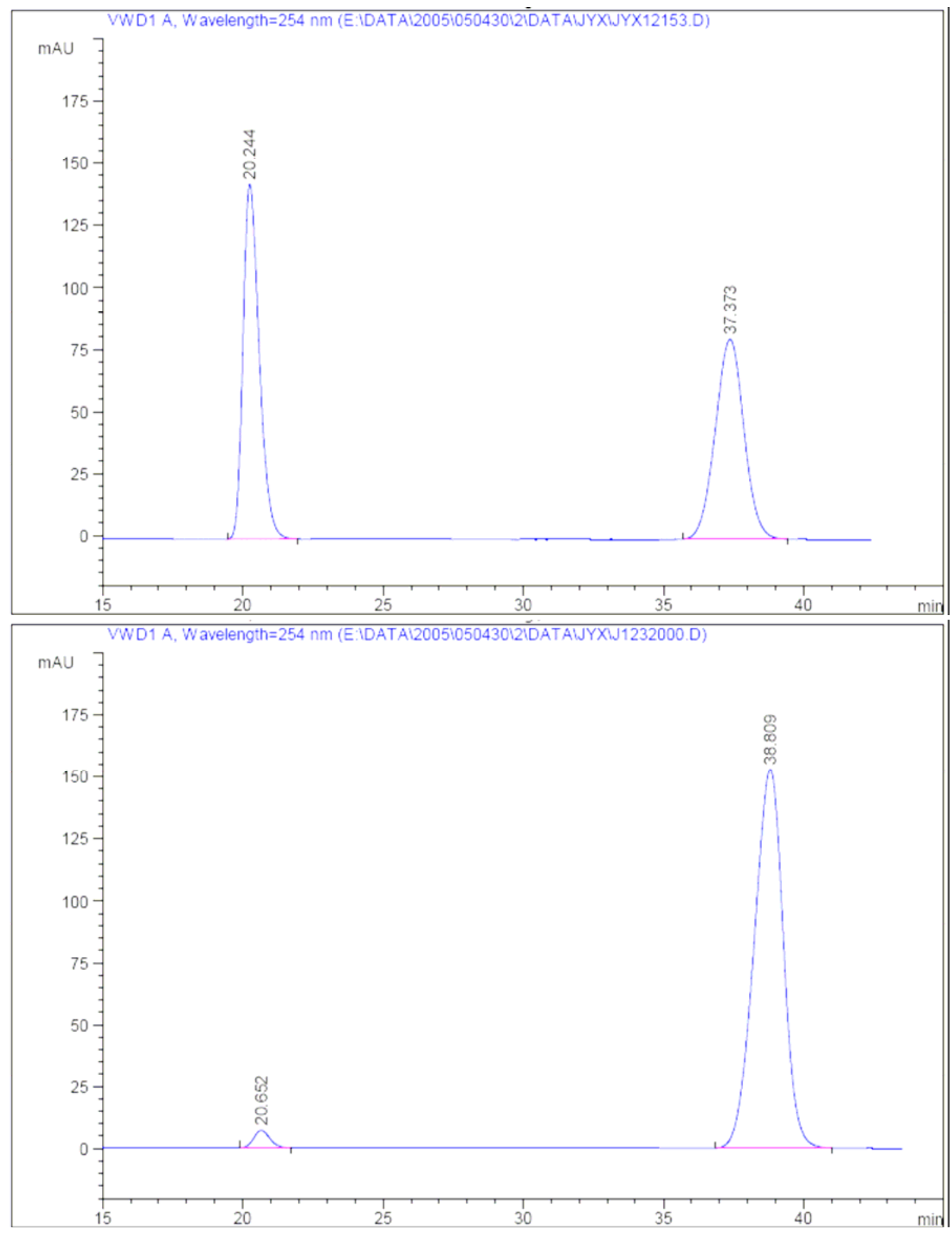

$N$-[Indol-3-yl-(2,6-dichlorophenyl)methyl]-4-methylbenzenesulfonamide (2j): white powder, 47\% yield, mp: $199-201^{\circ} \mathrm{C},[\alpha]^{20}{ }_{\mathrm{D}}=-217.2\left(\mathrm{c} 0.5, \mathrm{CH}_{2} \mathrm{Cl}_{2}\right), 93 \%$ ee [Daicel Chiralcel OD column $(25 \mathrm{~cm} \times 0.46 \mathrm{~cm} \mathrm{ID}), n$-hexane $/ \mathrm{i}-\mathrm{PrOH}=80: 20,1.0 \mathrm{~mL} / \mathrm{min}, 254 \mathrm{~nm} ; t_{\mathrm{R}}=11.49 \mathrm{~min}$ (minor) and $18.58 \mathrm{~min}] ;{ }^{1} \mathrm{H}$ NMR (300 MHz, $\left.\mathrm{CDCl}_{3}\right): \delta 2.29$ (s, 3H), $6.12(\mathrm{~d}, J=10.5 \mathrm{~Hz}, 1 \mathrm{H}), 6.73(\mathrm{~s}, 1 \mathrm{H})$, $6.79(\mathrm{~d}, J=10.5 \mathrm{~Hz}, 1 \mathrm{H}), 7.02-7.23(\mathrm{~m}, 5 \mathrm{H}), 7.20(\mathrm{t}, J=7.8 \mathrm{~Hz}, 2 \mathrm{H}), 7.34(\mathrm{~d}, J=8.1 \mathrm{~Hz}, 1 \mathrm{H}), 7.64$ $(\mathrm{d}, J=8.4 \mathrm{~Hz}, 3 \mathrm{H}), 8.05(\mathrm{~s}, 1 \mathrm{H}) ;{ }^{13} \mathrm{C}$ NMR $\left(75 \mathrm{MHz}, \mathrm{DMSO}-\mathrm{d}_{6}\right): \delta 20.8,50.9,110.3,111.6,118.2$, 
118.7, 121.2, 124.2, 125.3, 126.3, 129.0, 129.4, 134.3, 135.2, 136.2, 137.9, 142.3; ESI MS m/z 444 $\left(\mathrm{M}^{-}-1\right)$; Anal. Calcd. for $\mathrm{C}_{22} \mathrm{H}_{18} \mathrm{Cl}_{2} \mathrm{~N}_{2} \mathrm{O}_{2} \mathrm{~S}: \mathrm{C}, 59.33 ; \mathrm{H}, 4.07 ; \mathrm{N}, 6.29$. Found: $\mathrm{C}, 59.10 ; \mathrm{H}, 4.11 ; \mathrm{N}$, 6.04 .
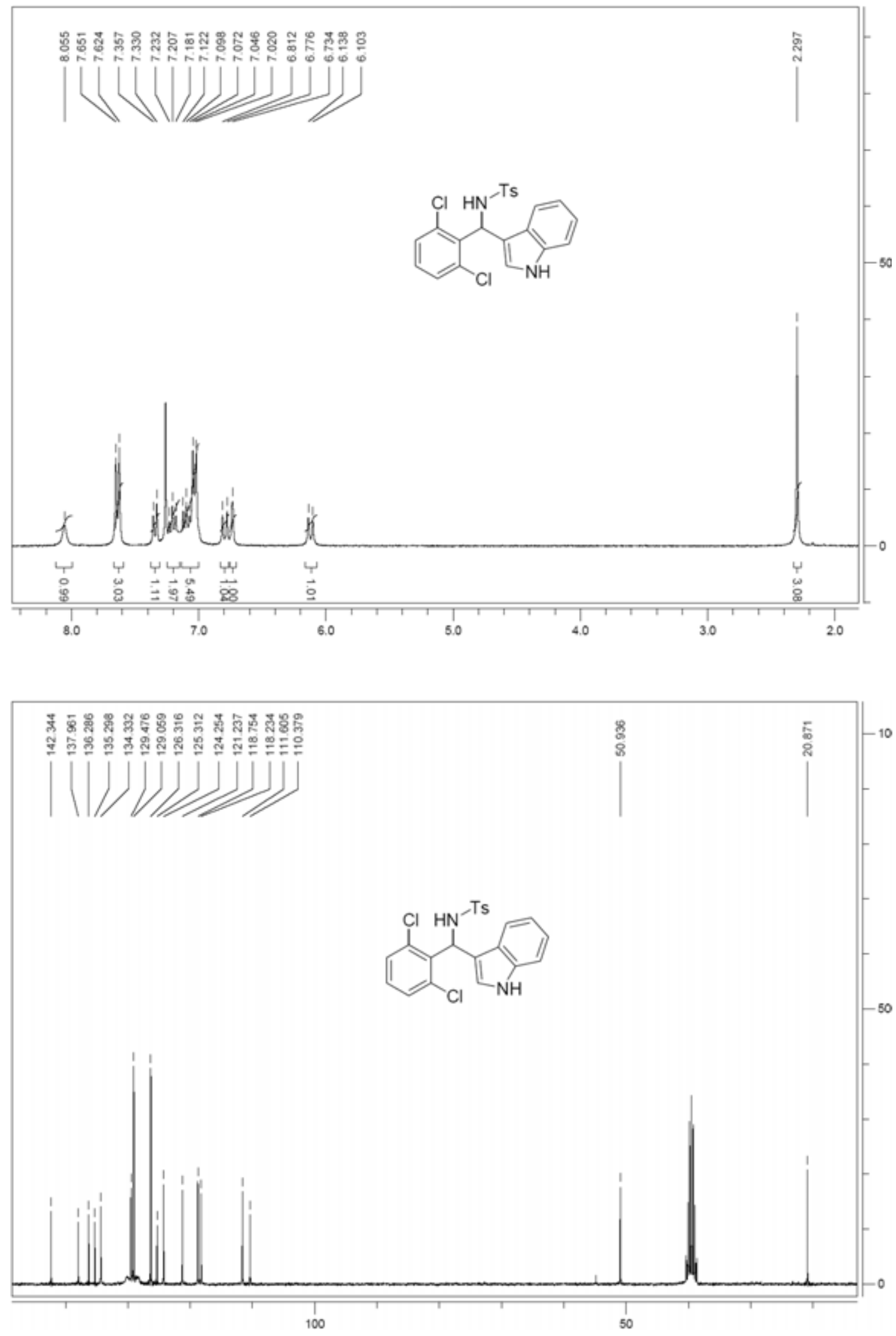

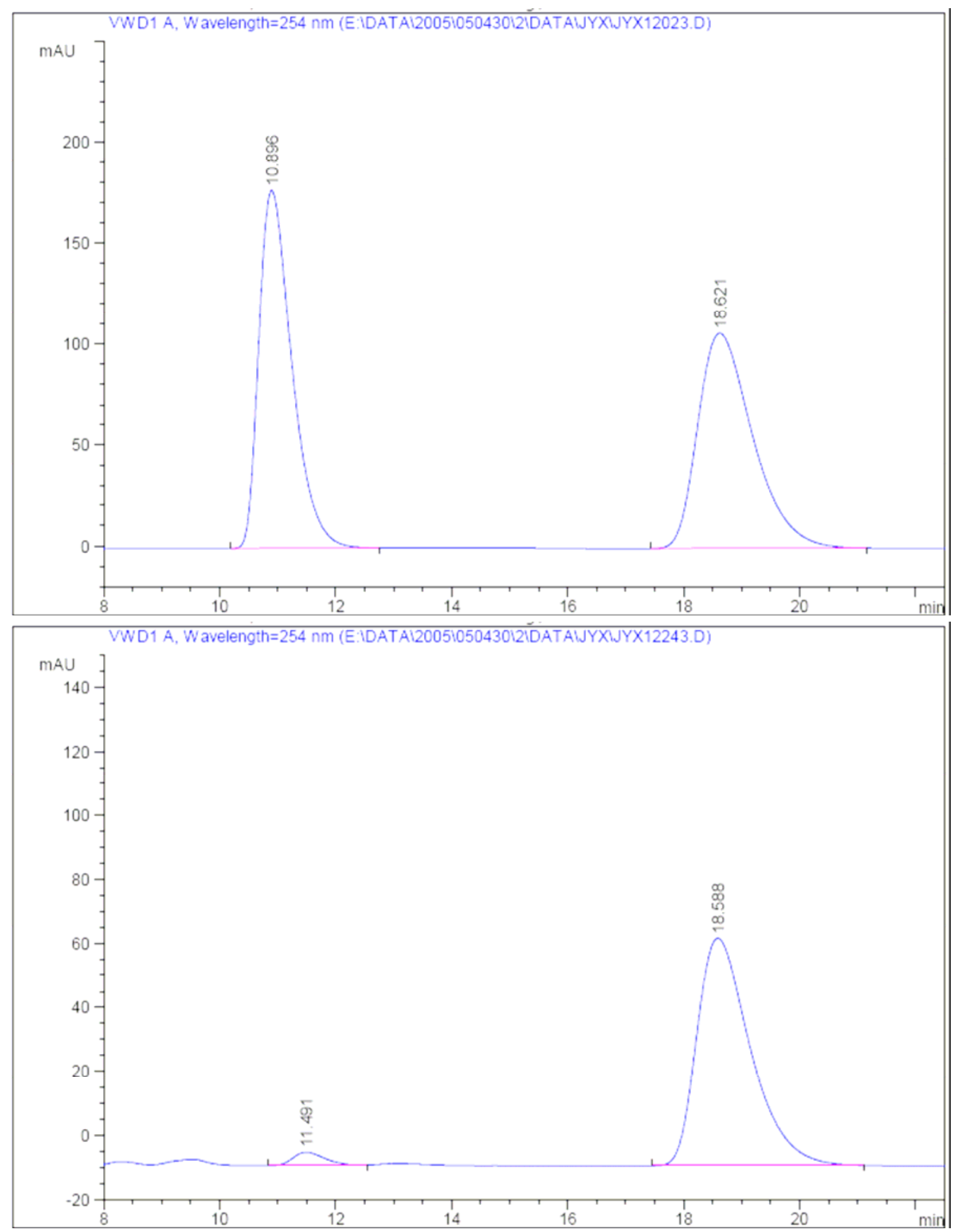

$N$-(Indol-3-yl-phenylmethyl)-4-nitrobenzenesulfonamide (2k): yellow powder, 86\% yield, mp: $164-166{ }^{\circ} \mathrm{C},[\alpha]^{20}{ }_{\mathrm{D}}=-14.9$ (c 1.4, AcOEt), 94\% ee [Daicel Chiralpak AD-H column $(25 \mathrm{~cm} \times 0.46$ $\mathrm{cm}$ ID), $n$-hexane/i-PrOH = 70:30, $1.0 \mathrm{~mL} / \mathrm{min}, 254 \mathrm{~nm} ; t_{\mathrm{R}}=15.32 \mathrm{~min}$ and $\left.23.77 \mathrm{~min}\right] ;{ }^{1} \mathrm{H}$ NMR (300 MHz, DMSO- $\left.d_{6}\right): \delta 5.86(\mathrm{~d}, J=8.7 \mathrm{~Hz}, 1 \mathrm{H}), 6.84(\mathrm{~d}, J=1.8 \mathrm{~Hz}, 1 \mathrm{H}), 6.91(\mathrm{t}, J=7.2 \mathrm{~Hz}, 1 \mathrm{H})$, $7.03(\mathrm{t}, J=7.2 \mathrm{~Hz}, 1 \mathrm{H}), 7.12-7.19(\mathrm{~m}, 3 \mathrm{H}), 7.28(\mathrm{t}, J=7.8 \mathrm{~Hz}, 3 \mathrm{H}), 7.44(\mathrm{~d}, J=7.8 \mathrm{~Hz}, 1 \mathrm{H}), 7.74$ $(\mathrm{d}, J=8.7 \mathrm{~Hz}, 2 \mathrm{H}), 8.02(\mathrm{~d}, J=8.7 \mathrm{~Hz}, 2 \mathrm{H}), 8.99(\mathrm{~d}, J=9.0 \mathrm{~Hz}, 1 \mathrm{H}), 10.92(\mathrm{~s}, 1 \mathrm{H}) ;{ }^{13} \mathrm{C}$ NMR $(75$ 
MHz, DMSO- $\left.d_{6}\right): \delta 54.8,111.3,114.7,118.6,118.9,121.2,123.3,124.0,125.2,126.8,127.1,127.7$, 127.9, 136.3, 140.6, 146.8, 148.5; ESI MS m/z $406\left(\mathrm{M}^{-}-1\right)$; Anal. Calcd. for $\mathrm{C}_{21} \mathrm{H}_{17} \mathrm{~N}_{3} \mathrm{O}_{4} \mathrm{~S}$ : C, 61.90; H, 4.21; N, 10.31. Found: C, 61.69; H, 4.31; N, 10.17 .
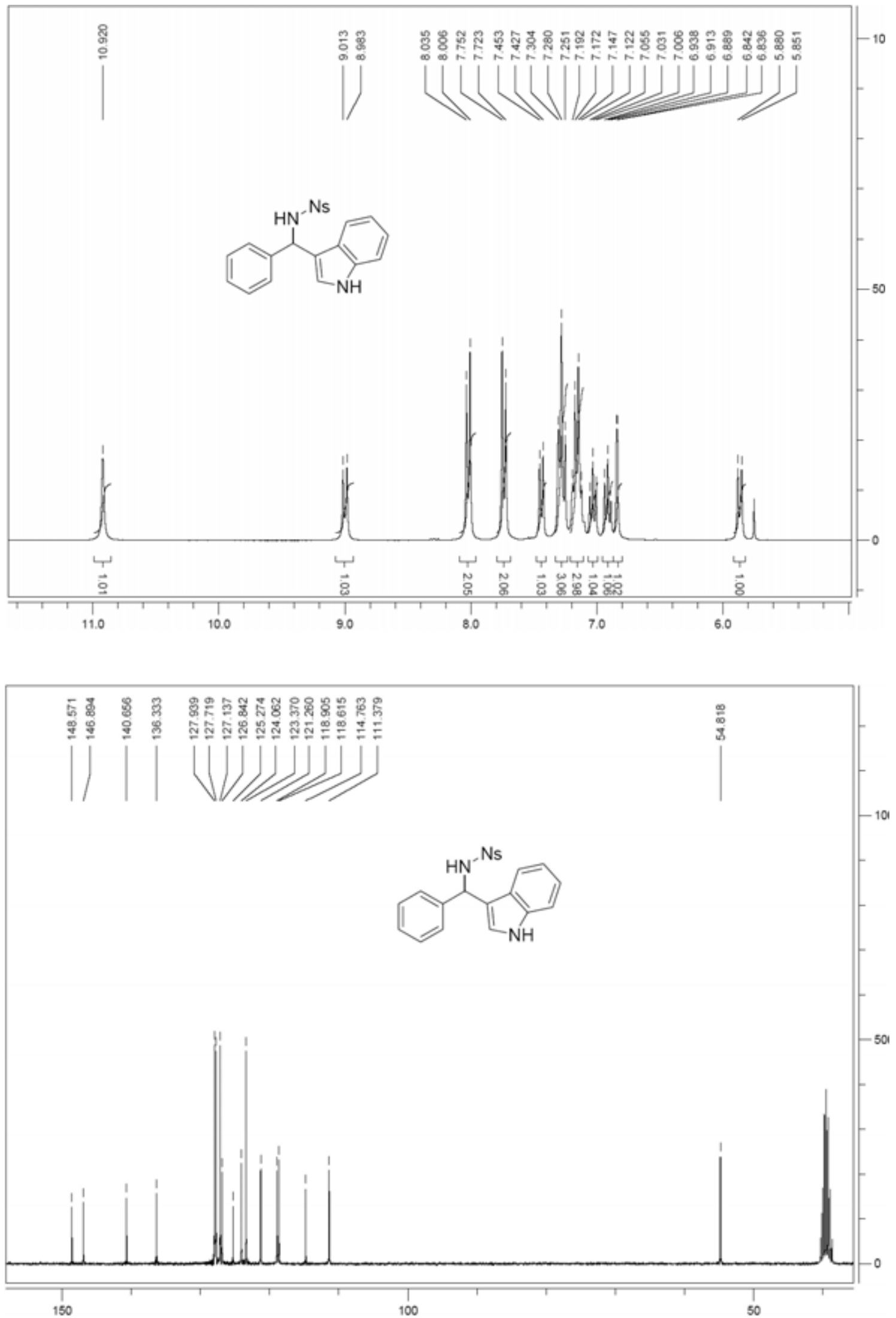

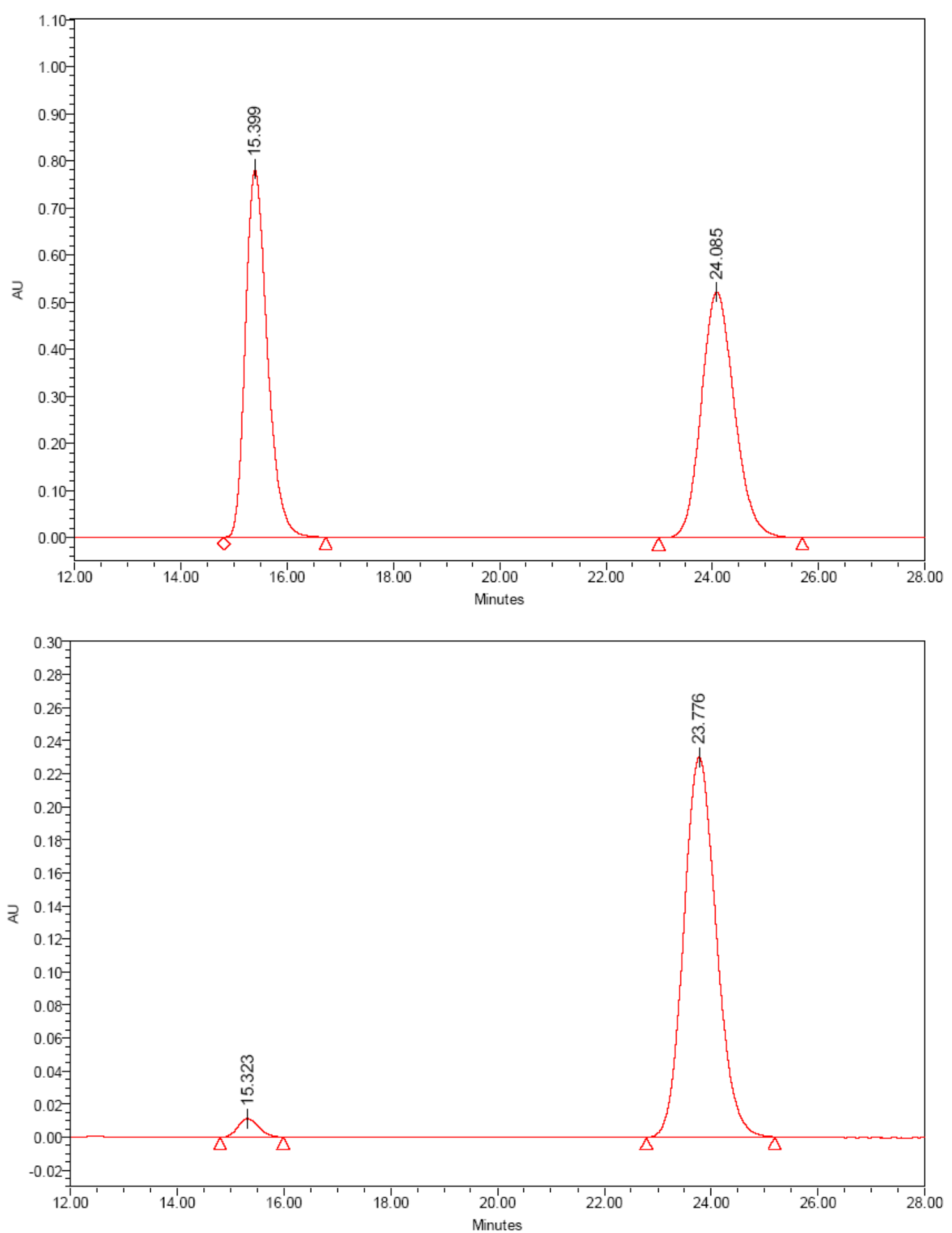

$N$-[Indol-3-yl-(4-fluorophenyl)methyl]-4-nitrobenzenesulfonamide (2l): yellow powder, 68\% yield, mp: $169-171^{\circ} \mathrm{C},[\alpha]^{20}{ }_{\mathrm{D}}=-16.8$ (c 1.1, AcOEt), 92\% ee [Daicel Chiralcel OD column $(25 \mathrm{~cm}$ $\times 0.46 \mathrm{~cm} \mathrm{ID}), n$-hexane $/ \mathrm{i}-\mathrm{PrOH}=70: 30,1.0 \mathrm{~mL} / \mathrm{min}, 254 \mathrm{~nm} ; t_{\mathrm{R}}=13.04 \mathrm{~min}$ and $\left.34.53 \mathrm{~min}\right] ;{ }^{1} \mathrm{H}$ NMR $\left(300 \mathrm{MHz}, \mathrm{CDCl}_{3}\right): \delta 5.36(\mathrm{~d}, J=7.2 \mathrm{~Hz}, 1 \mathrm{H}), 6.0(\mathrm{~d}, J=7.2 \mathrm{~Hz}, 1 \mathrm{H}), 6.72(\mathrm{~d}, J=2.7 \mathrm{~Hz}$, 1H), $6.92(\mathrm{t}, J=5.4 \mathrm{~Hz}, 2 \mathrm{H}), 7.04(\mathrm{t}, J=7.8 \mathrm{~Hz}, 1 \mathrm{H}), 7.17$ (t, $J=7.8 \mathrm{~Hz}, 1 \mathrm{H}), 7.25(\mathrm{dd}, \mathrm{J}=2.7,8.1$ Hz, 4H), 7.33 (d, $J=8.1 \mathrm{~Hz}, 1 \mathrm{H}), 7.67$ (d, $J=8.4 \mathrm{~Hz}, 1 \mathrm{H}), 7.98(\mathrm{~d}, J=8.4 \mathrm{~Hz}, 2 \mathrm{H}), 8.02(\mathrm{~s}, 1 \mathrm{H})$; 
${ }^{13} \mathrm{C}$ NMR (75 MHz, DMSO-d $): \delta 54.1,111.4,114.5,114.7,118.6,118.8,121.3,123.4,124.0$, 125.1, 127.7, 129.0, 129.1, 136.3, 136.8, 146.8, 148.6; ESI MS m/z 424 (M-1); Anal. Calcd. for $\mathrm{C}_{21} \mathrm{H}_{16} \mathrm{FN}_{3} \mathrm{O}_{4} \mathrm{~S}: \mathrm{C}, 59.29 ; \mathrm{H}, 3.79 ; \mathrm{N}, 9.88$. Found: C, 59.52; H, 3.67; N, 10.15 .
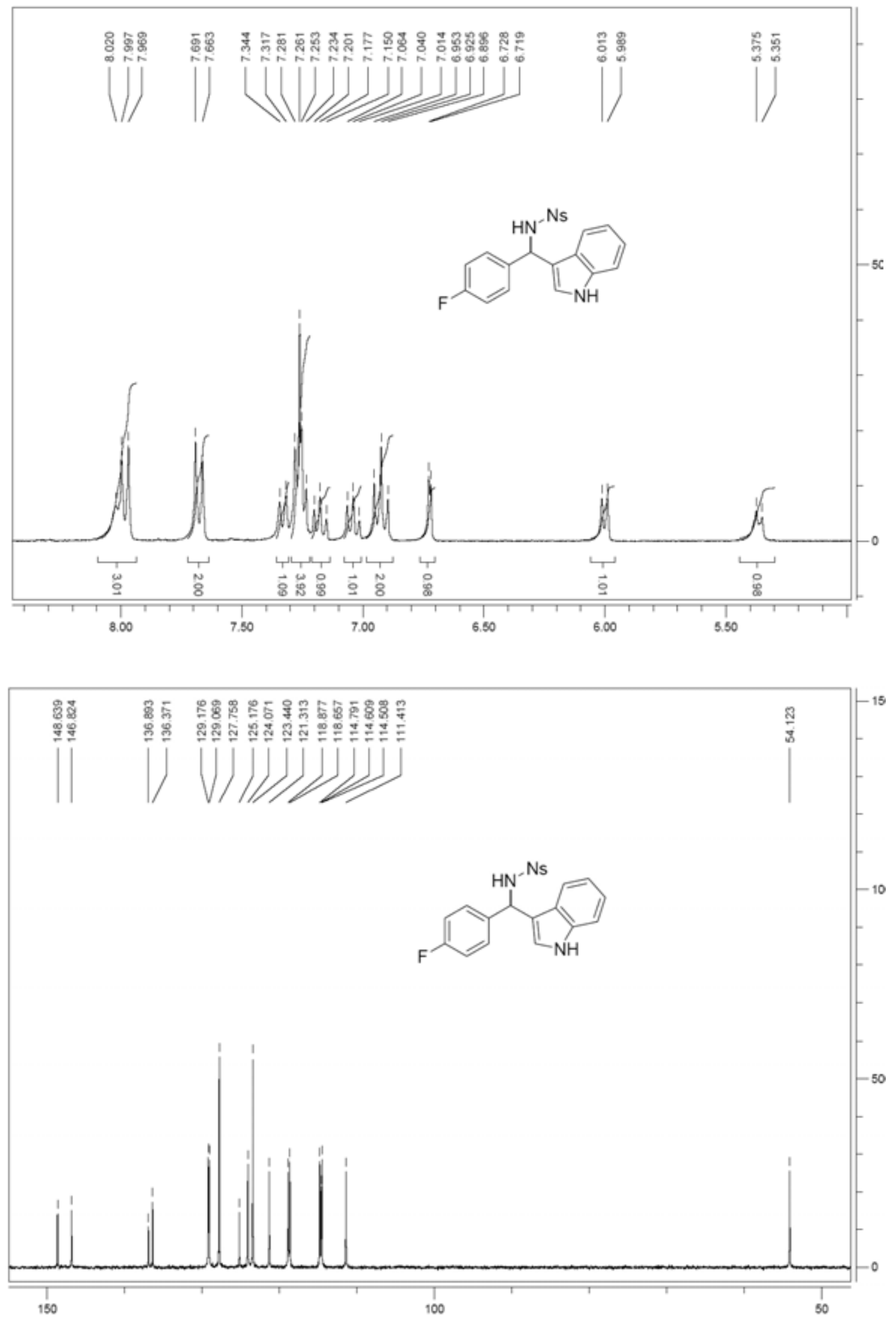

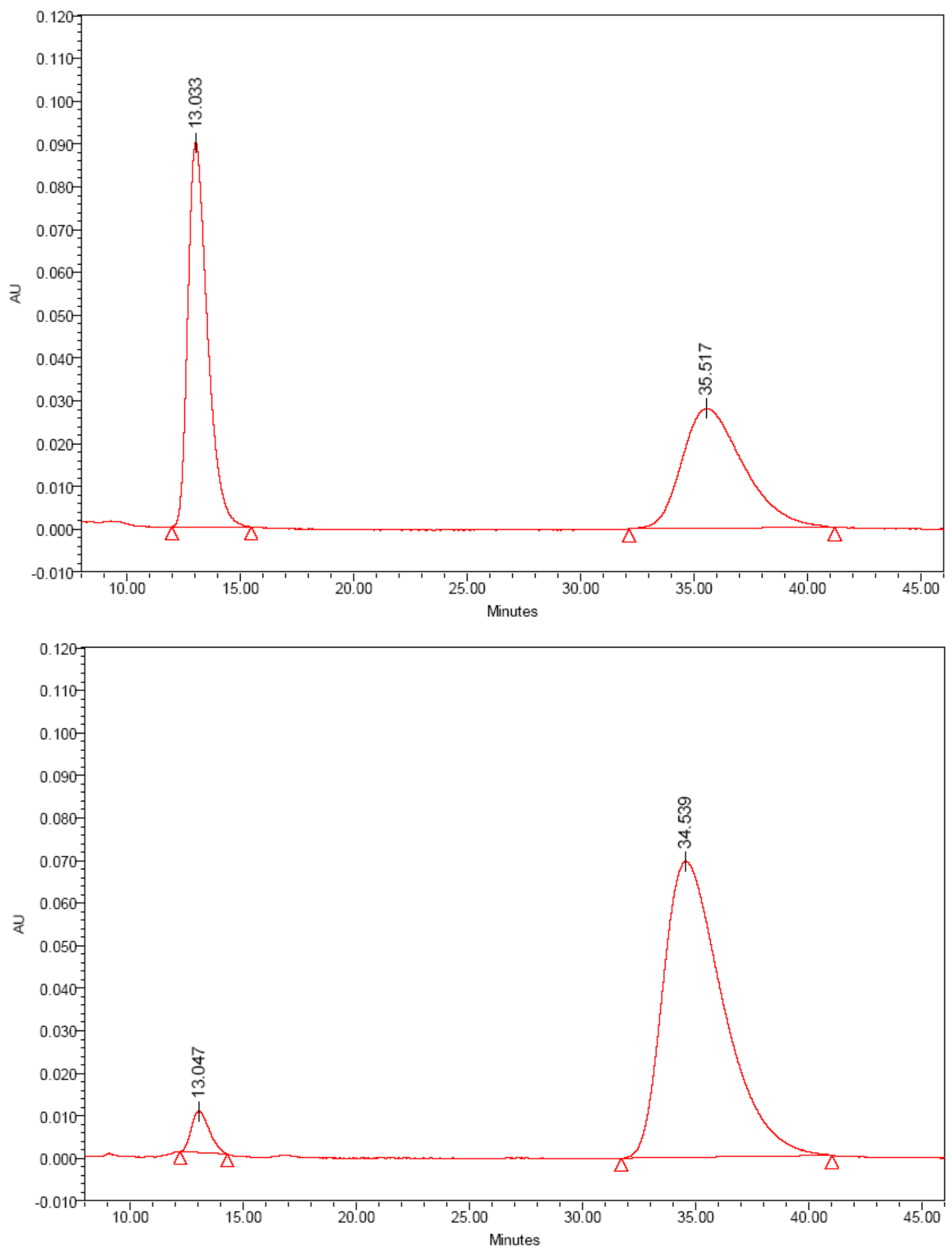

$N$-[Indol-3-yl-(4-methylphenyl)methyl]-4-nitrobenzenesulfonamide (2m): yellow powder, 84\% yield, mp: $158-160{ }^{\circ} \mathrm{C},[\alpha]^{20}{ }_{\mathrm{D}}=-17.3$ (c 1.0, AcOEt), 94\% ee [Daicel Chiralpak AD-H column $(25 \mathrm{~cm} \times 0.46 \mathrm{~cm} \mathrm{ID}), n$-hexane $/ \mathrm{i}-\mathrm{PrOH}=70: 30,1.0 \mathrm{~mL} / \mathrm{min}, 254 \mathrm{~nm} ; t_{\mathrm{R}}=11.44 \mathrm{~min}$ and $25.24 \mathrm{~min}] ;{ }^{1} \mathrm{H}$ NMR $\left(300 \mathrm{MHz}, \mathrm{CDCl}_{3}\right): \delta 2.27$ (s, 3H), $5.35(\mathrm{~d}, J=6.3 \mathrm{~Hz}, 1 \mathrm{H}), 5.98(\mathrm{~d}, J=6.3$ $\mathrm{Hz}, 1 \mathrm{H}), 6.72(\mathrm{~s}, 1 \mathrm{H}), 6.97-7.25(\mathrm{~m}, 7 \mathrm{H}), 7.45(\mathrm{~d}, J=7.5 \mathrm{~Hz}, 1 \mathrm{H}), 7.63(\mathrm{~d}, J=8.4 \mathrm{~Hz}, 2 \mathrm{H}), 7.92(\mathrm{~d}$, $J=8.4 \mathrm{~Hz}, 2 \mathrm{H}), 7.98(\mathrm{~s}, 1 \mathrm{H}) ;{ }^{13} \mathrm{C}$ NMR $\left(75 \mathrm{MHz}, \mathrm{DMSO}-d_{6}\right): \delta 20.3,54.5,111.3,114.7,118.6$, 
$118.8,121.2,123.2,123.7,125.1,127.0,127.7,128.4,136.1,137.4,146.7,148.4$; ESI MS m/z 420 $\left(\mathrm{M}^{-}-1\right)$; Anal. Calcd. for $\mathrm{C}_{22} \mathrm{H}_{19} \mathrm{~N}_{3} \mathrm{O}_{4} \mathrm{~S}$ : C, 62.69; H, 4.54; N, 9.97. Found: $\mathrm{C}, 62.97 ; \mathrm{H}, 4.39 ; \mathrm{N}$, 10.14 .
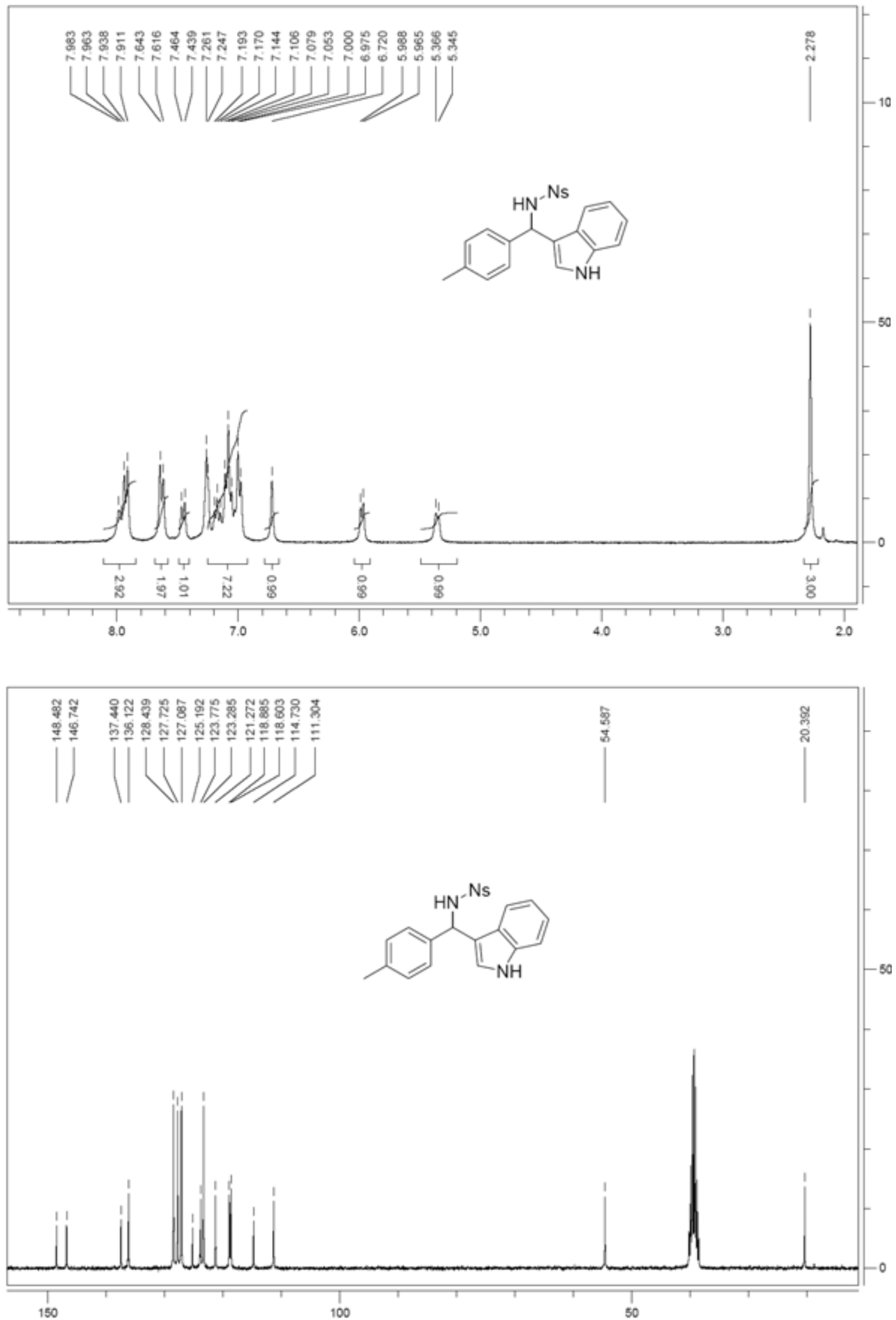

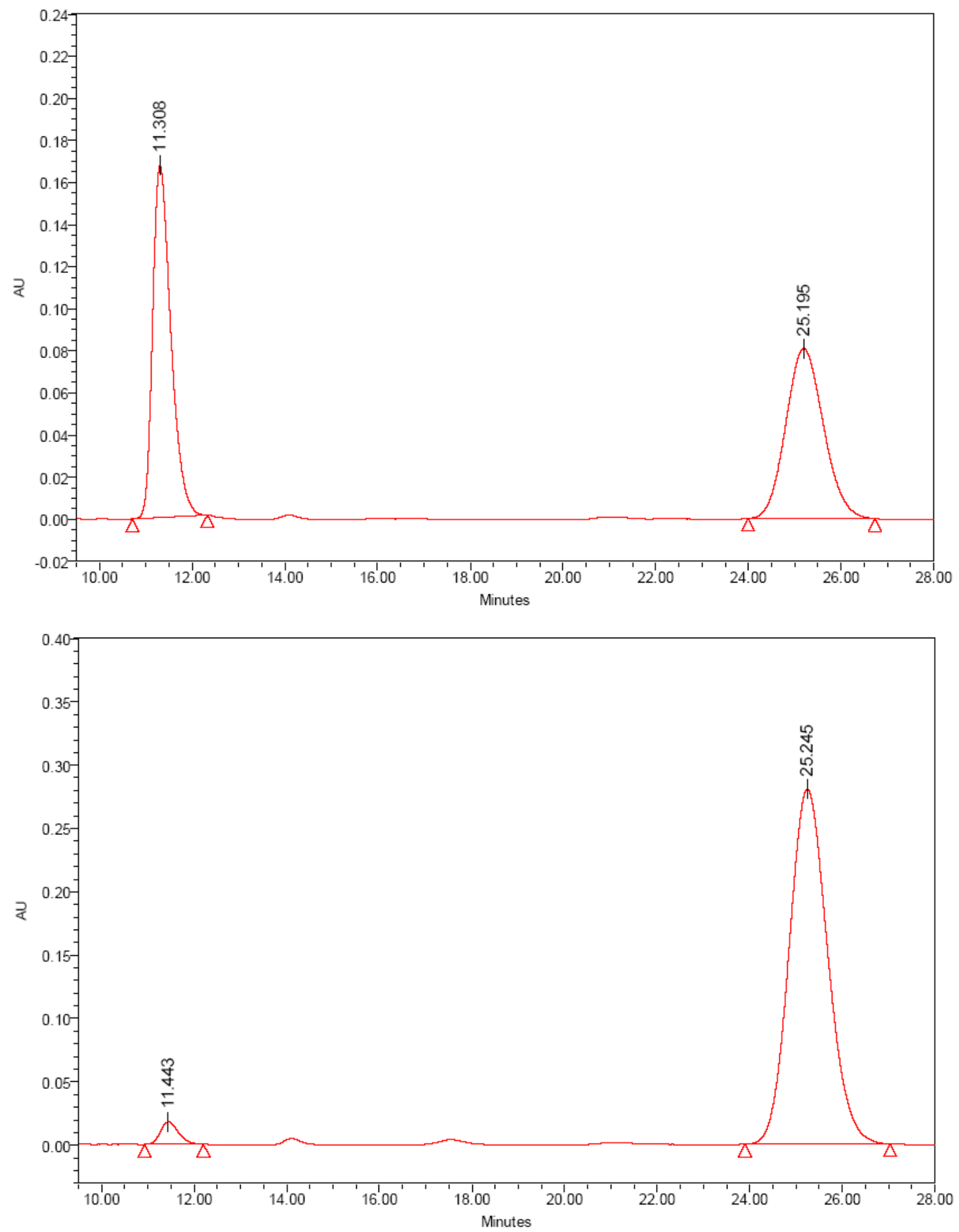

$N$-[Indol-3-yl-(3-methylphenyl)methyl]-4-nitrobenzenesulfonamide (2n): yellow powder, 91\% yield, mp: $160-161{ }^{\circ} \mathrm{C},[\alpha]^{20}{ }_{\mathrm{D}}=-12.9$ ( c 1.8 , AcOEt), 95\% ee [Daicel Chiralpak AD-H column $(25 \mathrm{~cm} \times 0.46 \mathrm{~cm} \mathrm{ID}), n$-hexane $/ \mathrm{i}-\mathrm{PrOH}=70: 30,1.0 \mathrm{~mL} / \mathrm{min}, 254 \mathrm{~nm} ; t_{\mathrm{R}}=12.22 \mathrm{~min}$ and $18.57 \mathrm{~min}$ ]; ${ }^{1} \mathrm{H}$ NMR (300 MHz, $\left.\mathrm{CDCl}_{3}\right): \delta 2.18$ (s, 3H), $5.39(\mathrm{~d}, J=7.2 \mathrm{~Hz}, 1 \mathrm{H}), 5.98(\mathrm{~d}, J=7.2$ $\mathrm{Hz}, 1 \mathrm{H}), 6.70(\mathrm{~d}, J=2.7 \mathrm{~Hz}, 1 \mathrm{H}), 6.98-7.10(\mathrm{~m}, 5 \mathrm{H}), 7.18(\mathrm{t}, J=7.8 \mathrm{~Hz}, 1 \mathrm{H}), 7.27$ (d, $J=5.4 \mathrm{~Hz}$ 1H), 7.49 (d, $J=7.8 \mathrm{~Hz}, 1 \mathrm{H}), 7.63(\mathrm{~d}, J=8.7 \mathrm{~Hz}, 2 \mathrm{H}), 7.93(\mathrm{~d}, J=8.7 \mathrm{~Hz}, 2 \mathrm{H}), 7.98(\mathrm{~s}, 1 \mathrm{H}) ;{ }^{13} \mathrm{C}$ NMR (75 MHz, DMSO-d $\left.{ }_{6}\right): \delta 20.8,54.8,111.3,114.7,118.6,118.8,121.2,123.2,124.0,124.3$, 
125.3, 127.3, 127.7, 127.8, 136.3, 136.9, 140.3, 146.9, 148.5; ESI MS m/z 420 (M-1); Anal. Calcd. for $\mathrm{C}_{22} \mathrm{H}_{19} \mathrm{~N}_{3} \mathrm{O}_{4} \mathrm{~S}$ : C, 62.69; H, 4.54; N, 9.97. Found: C, 62.91; H, 4.67; N, 9.85.
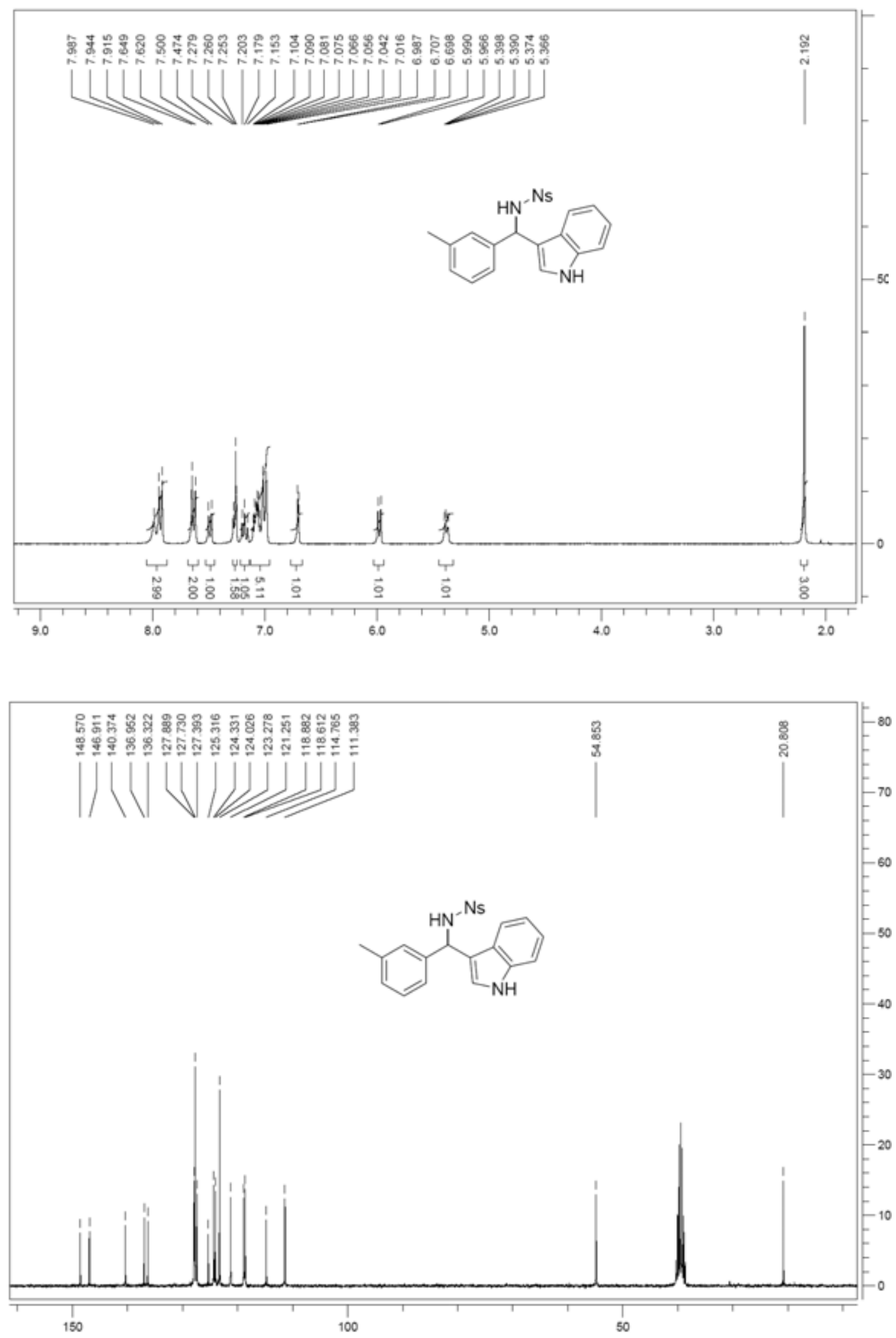

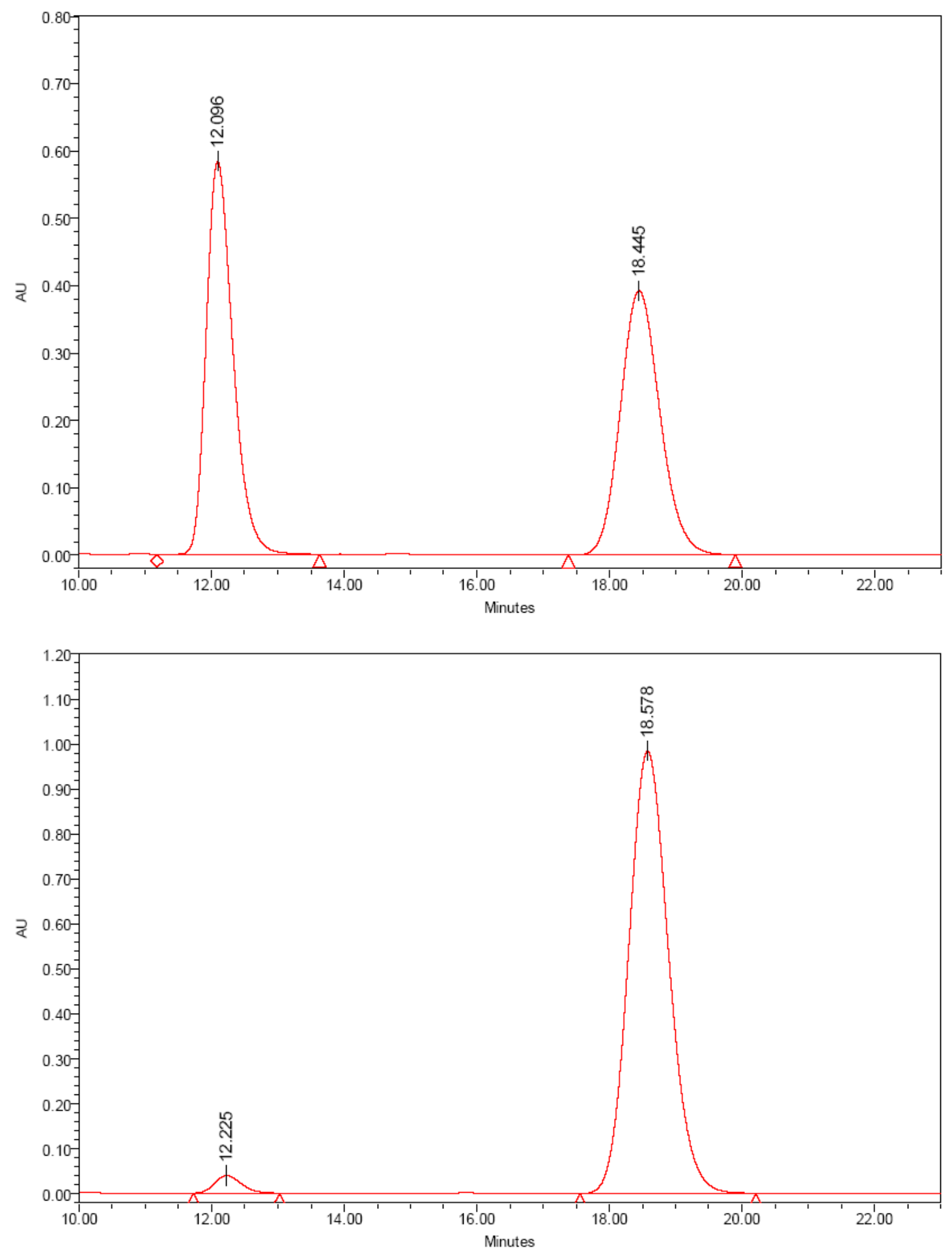

$N$-[Indol-3-yl-(2-methylphenyl)methyl]-4-nitrobenzenesulfonamide (2o): yellow powder, $63 \%$ yield, mp: $183-184{ }^{\circ} \mathrm{C},[\alpha]^{20}{ }_{\mathrm{D}}=-6.1$ (c 1.0, AcOEt), $89 \%$ ee [Daicel Chiralpak AD-H column $(25 \mathrm{~cm} \times 0.46 \mathrm{~cm} \mathrm{ID}), n$-hexane $/ \mathrm{i}-\mathrm{PrOH}=70: 30,1.0 \mathrm{~mL} / \mathrm{min}, 254 \mathrm{~nm} ; t_{\mathrm{R}}=13.87 \mathrm{~min}$ and 15.61 min]; ${ }^{1} \mathrm{H}$ NMR (300 MHz, $\left.\mathrm{CDCl}_{3}\right): \delta 2.30(\mathrm{~s}, 3 \mathrm{H}), 5.28(\mathrm{t}, J=7.5 \mathrm{~Hz}, 1 \mathrm{H}), 6.27(\mathrm{~d}, J=6.6 \mathrm{~Hz}, 1 \mathrm{H})$, $6.56(\mathrm{~s}, 1 \mathrm{H}), 6.89-6.94(\mathrm{~m}, 1 \mathrm{H}), 7.07-7.15(\mathrm{~m}, 4 \mathrm{H}), 7.19-7.32(\mathrm{~m}, 2 \mathrm{H}), 7.63-7.66(\mathrm{~m}, 3 \mathrm{H})$, 7.94-7.98 (m, 3H); ${ }^{13} \mathrm{C}$ NMR $\left(75 \mathrm{MHz}, \mathrm{DMSO}-d_{6}\right): \delta 18.6,51.0,111.44,111.49,113.6,118.5$, 
118.7, 121.3, 123.4, 124.3, 124.4, 125.5, 126.8, 127.5, 129.9, 134.6, 136.2, 138.4, 146.7, 148.6; HRMS (ESI) Calcd. for $\mathrm{C}_{22} \mathrm{H}_{23} \mathrm{~N}_{4} \mathrm{O}_{4} \mathrm{~S}\left(\mathrm{M}+\mathrm{NH}_{4}\right): 439.1435$. Found: 439.1439 .
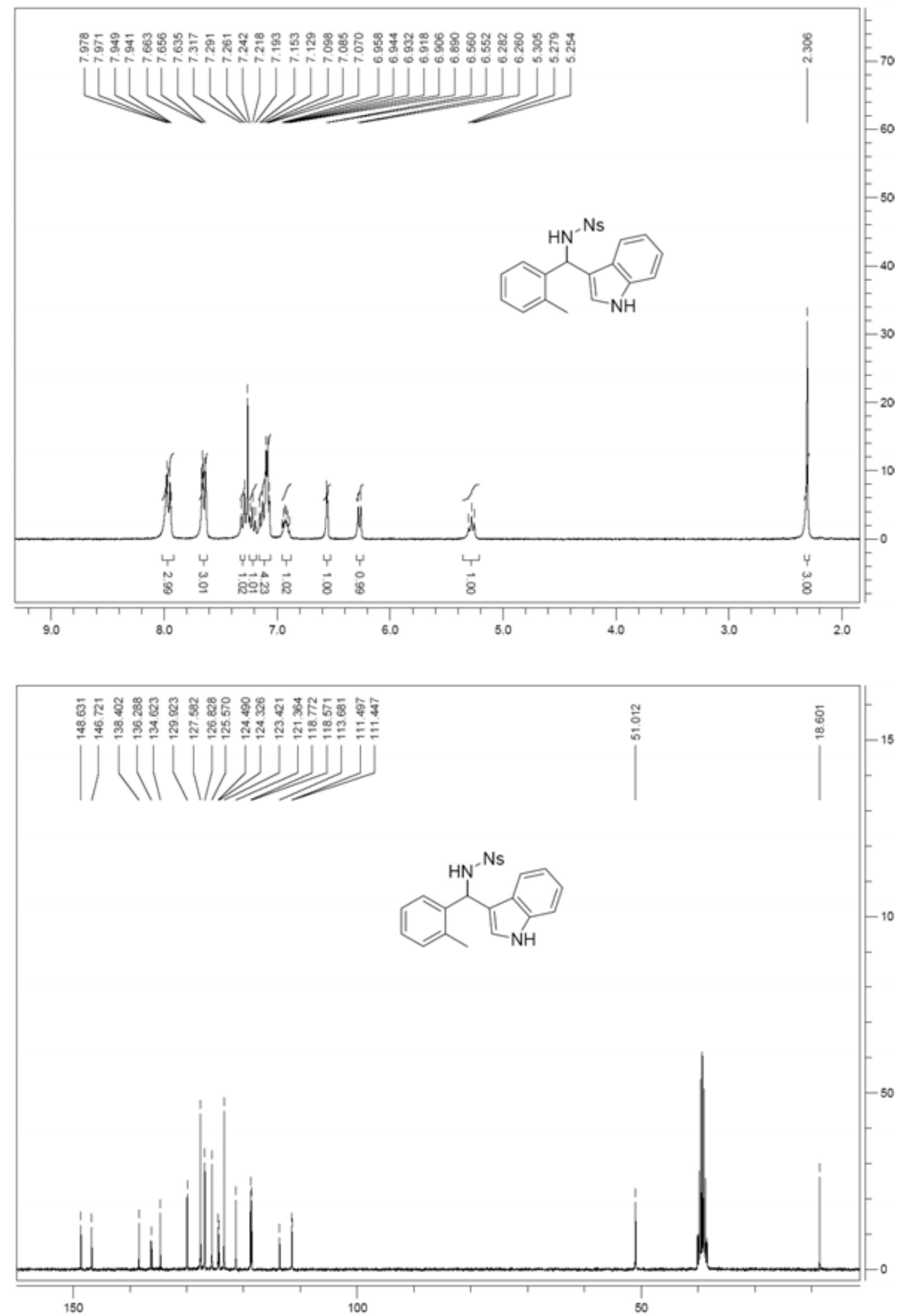

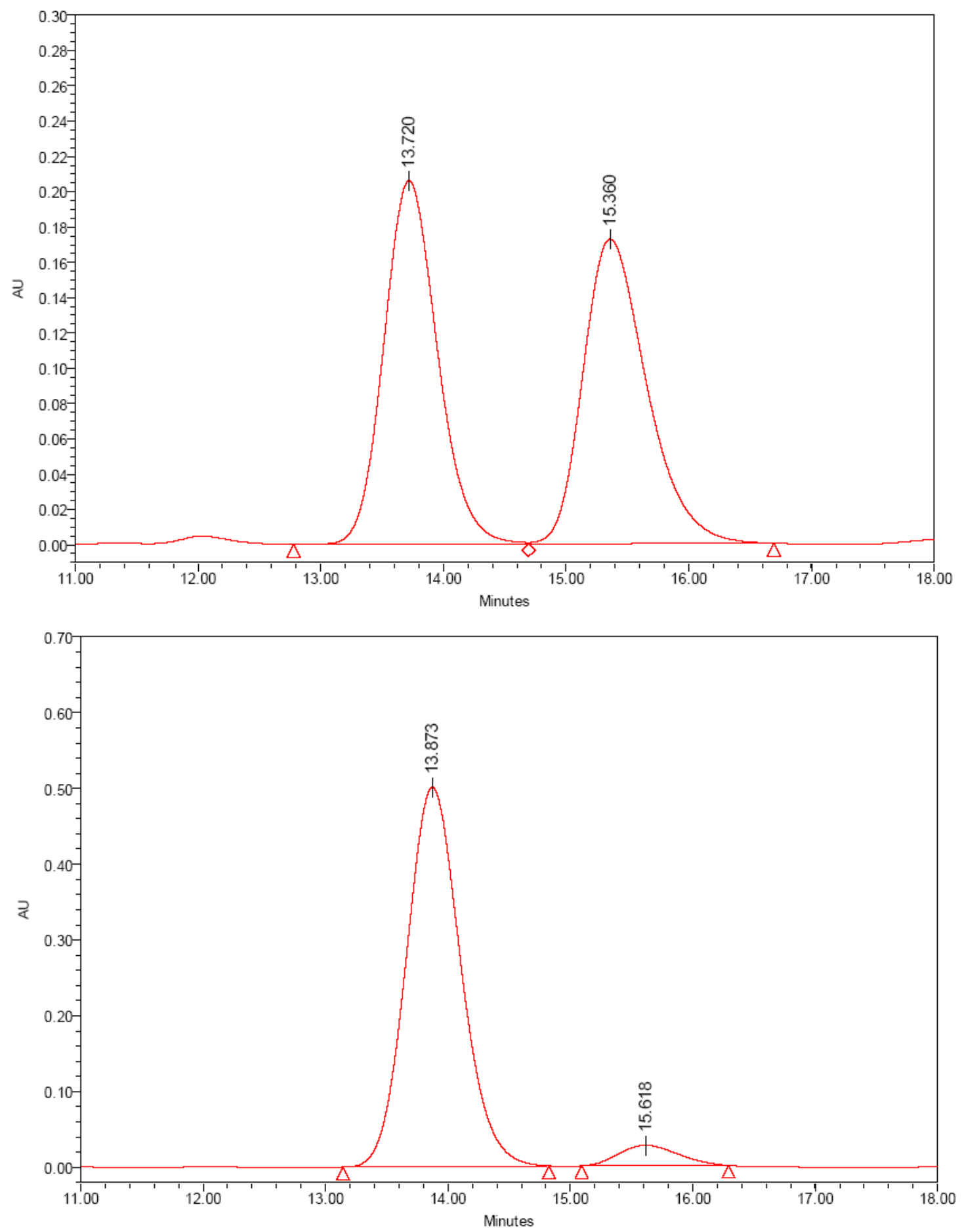

$N$-[Indol-3-yl-(4-methoxyphenyl)methyl]-4-nitrobenzenesulfonamide (2p): yellow powder, 47\% yield, mp: $163-165{ }^{\circ} \mathrm{C},[\alpha]^{20}{ }_{\mathrm{D}}=-6.7$ (c 0.7, AcOEt), 88\% ee [Daicel Chiralpak AD-H column $(25 \mathrm{~cm} \times 0.46 \mathrm{~cm} \mathrm{ID}), n$-hexane $/ \mathrm{i}-\mathrm{PrOH}=70: 30,1.0 \mathrm{~mL} / \mathrm{min}, 254 \mathrm{~nm} ; t_{\mathrm{R}}=13.51 \mathrm{~min}$ and 31.07 min]; ${ }^{1} \mathrm{H}$ NMR (300 MHz, $\left.\mathrm{CDCl}_{3}\right): \delta 3.74(\mathrm{~s}, 3 \mathrm{H}), 5.28$ (d, J=6.3 Hz, 1H), 5.97 (d, J=6.3 Hz, 1H), $6.70(\mathrm{~s}, 1 \mathrm{H}), 6.74(\mathrm{t}, J=3.3 \mathrm{~Hz}, 2 \mathrm{H}), 7.04(\mathrm{t}, J=7.2 \mathrm{~Hz}, 1 \mathrm{H}), 7.12-7.19(\mathrm{~m}, 3 \mathrm{H}), 7.26(\mathrm{t}, J=4.2 \mathrm{~Hz}$ 2H), $7.65(\mathrm{~d}, J=8.7 \mathrm{~Hz}, 2 \mathrm{H}), 7.96(\mathrm{~d}, J=8.7 \mathrm{~Hz}, 2 \mathrm{H}) \mathrm{m}, 7.99(\mathrm{~s}, 1 \mathrm{H}) ;{ }^{13} \mathrm{C} \mathrm{NMR}(75 \mathrm{MHz}$, DMSO- $\left.d_{6}\right): \delta 54.3,54.9,111.3,113.2,114.9,118.6,118.9,121.2,123.3,123.7,125.2,127.7,128.3$, 
132.5, 136.3, 146.8, 148.5, 158.2; ESI MS m/z $436\left(\mathrm{M}^{-}-1\right)$; Anal. Calcd. for $\mathrm{C}_{22} \mathrm{H}_{19} \mathrm{~N}_{3} \mathrm{O}_{5} \mathrm{~S}$ : C, 60.40; H, 4.38; N, 9.61. Found: C, 60.24; H, 4.21; N, 9.83.
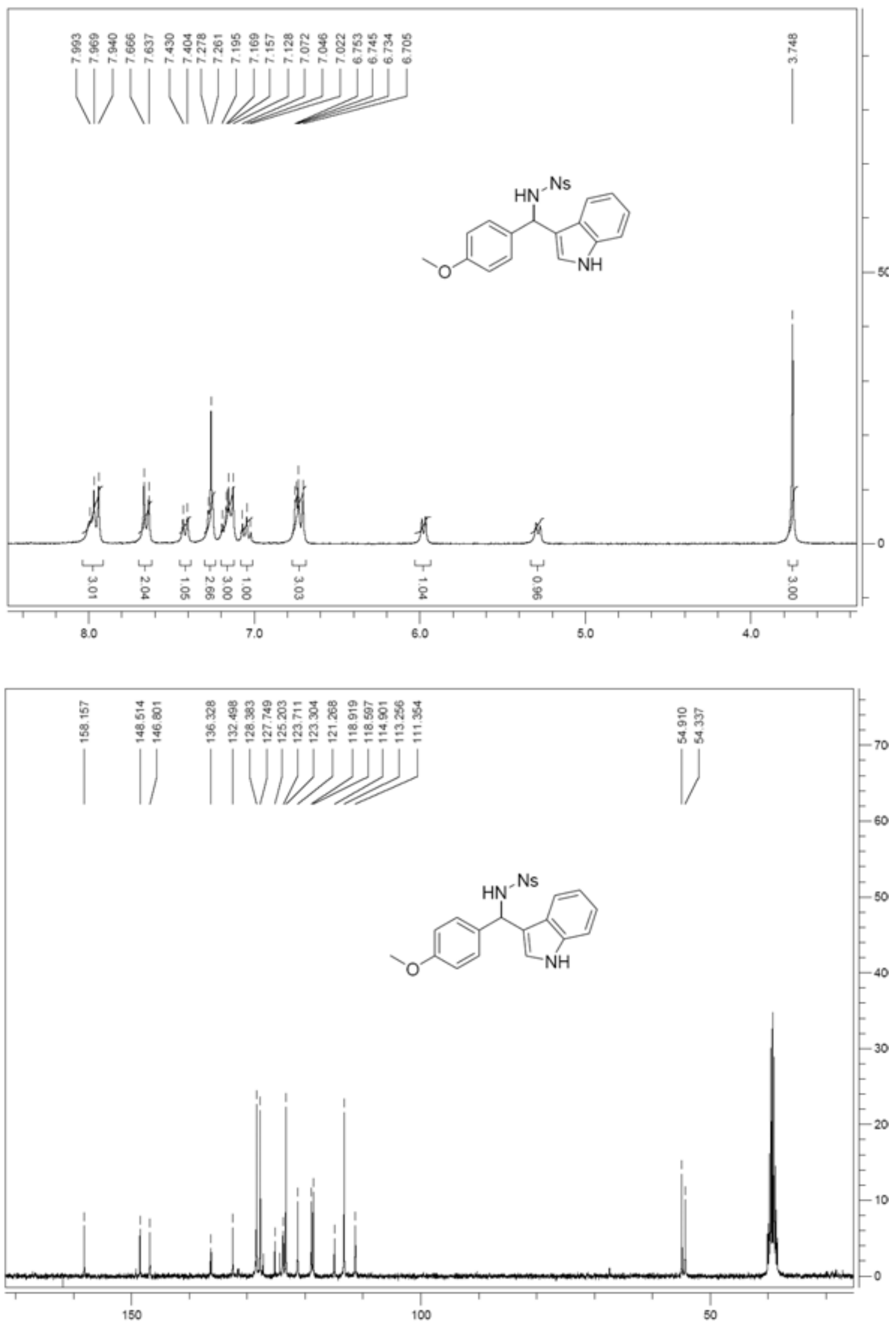

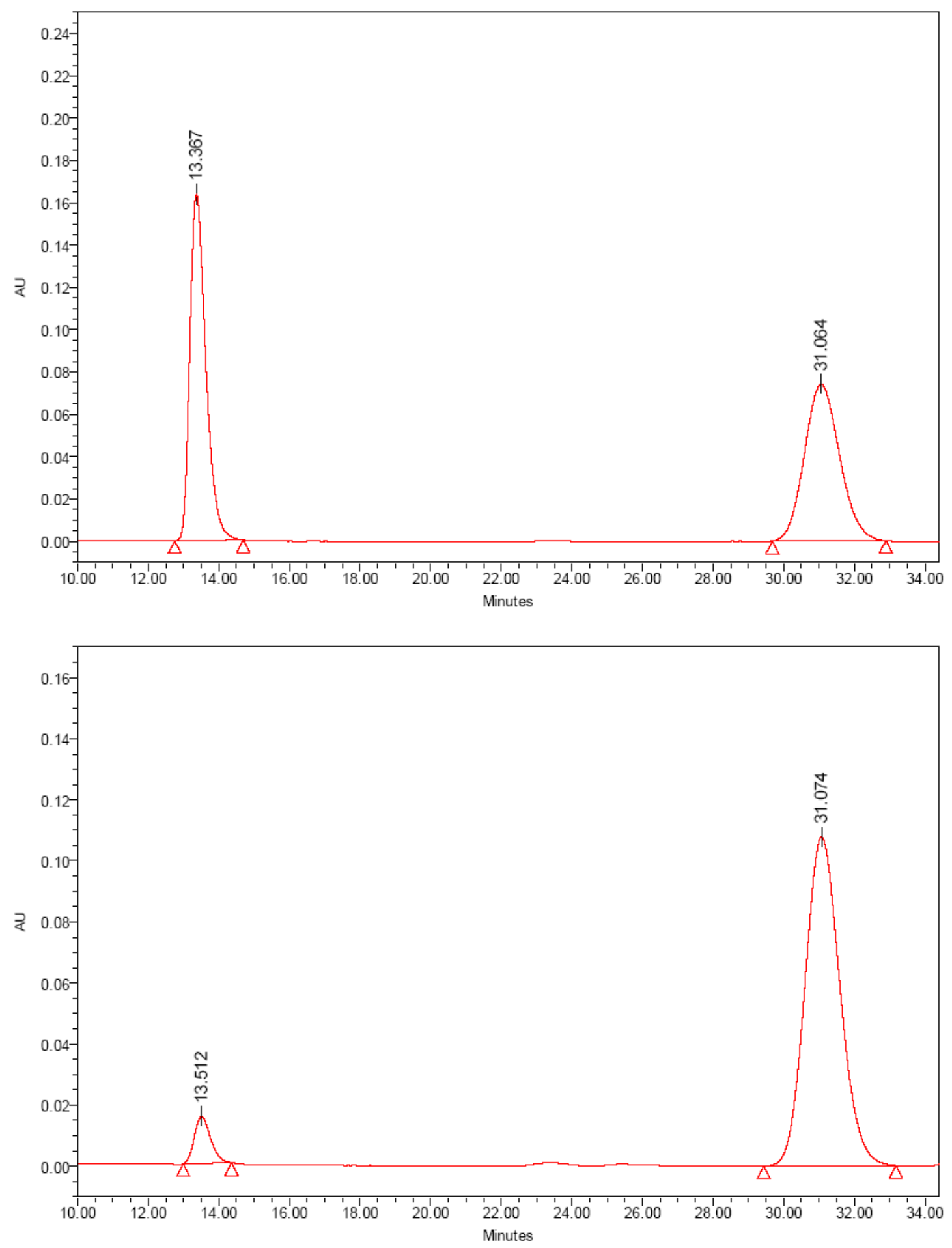

$N$-[Indol-3-yl-(1-naphthyl)methyl]-4-nitrobenzenesulfonamide (2q): yellow powder，85\% yield, mp: $160-162{ }^{\circ} \mathrm{C},[\alpha]^{20}{ }_{\mathrm{D}}=-36.1$ (c 1.6, AcOEt), 81\% ee [Daicel Chiralpak AD-H column $(25$ $\mathrm{cm} \times 0.46 \mathrm{~cm} \mathrm{ID}), n$-hexane $/ \mathrm{i}-\mathrm{PrOH}=70: 30,1.0 \mathrm{~mL} / \mathrm{min}, 254 \mathrm{~nm} ; t_{\mathrm{R}}=30.47 \mathrm{~min}$ and $\left.38.14 \mathrm{~min}\right]$; ${ }^{1} \mathrm{H}$ NMR $\left(300 \mathrm{MHz}, \mathrm{DMSO}-d_{6}\right): \delta 6.65(\mathrm{~s}, 1 \mathrm{H}), 6.81(\mathrm{~s}, 1 \mathrm{H}), 6.91(\mathrm{t}, J=6.6 \mathrm{~Hz}, 1 \mathrm{H}), 7.04(\mathrm{t}, J=$ $6.6 \mathrm{~Hz}, 1 \mathrm{H}), 7.25-7.36(\mathrm{~m}, 2 \mathrm{H}), 7.48-7.56(\mathrm{~m}, 4 \mathrm{H}), 7.66(\mathrm{~d}, J=7.5 \mathrm{~Hz}, 2 \mathrm{H}), 7.77$ (d, $J=8.4 \mathrm{~Hz}$, 1H), 7.86-7.93 (m, 3H), $8.16(\mathrm{~d}, J=6.9 \mathrm{~Hz}, 1 \mathrm{H}), 9.14(\mathrm{~s}, 1 \mathrm{H}), 10.93(\mathrm{~s}, 1 \mathrm{H}) ;{ }^{13} \mathrm{C} \mathrm{NMR}(75 \mathrm{MHz}$, 
DMSO-d $)_{6}: \delta 51.4,111.4,113.9,118.7,121.3,123.1,123.3,124.8,124.9,125.3,125.5,126.2$, 127.4, 127.7, 128.4, 130.2, 133.2, 135.9, 136.3, 146.6, 148.4; HRMS (EI) Calcd. for $\mathrm{C}_{25} \mathrm{H}_{19} \mathrm{~N}_{3} \mathrm{O}_{4} \mathrm{~S}$ (M): 457.1096. Found: 457.1094.
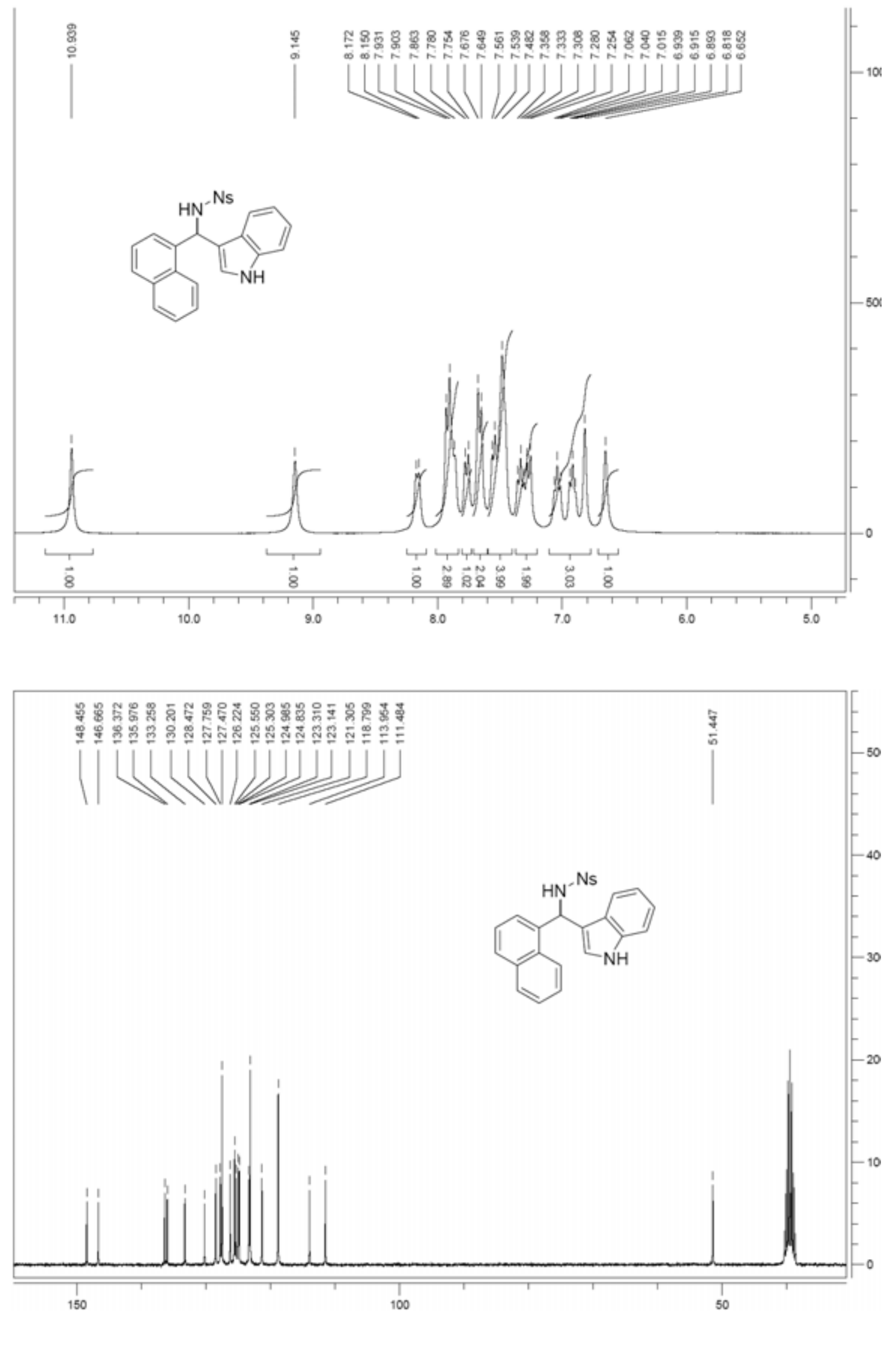

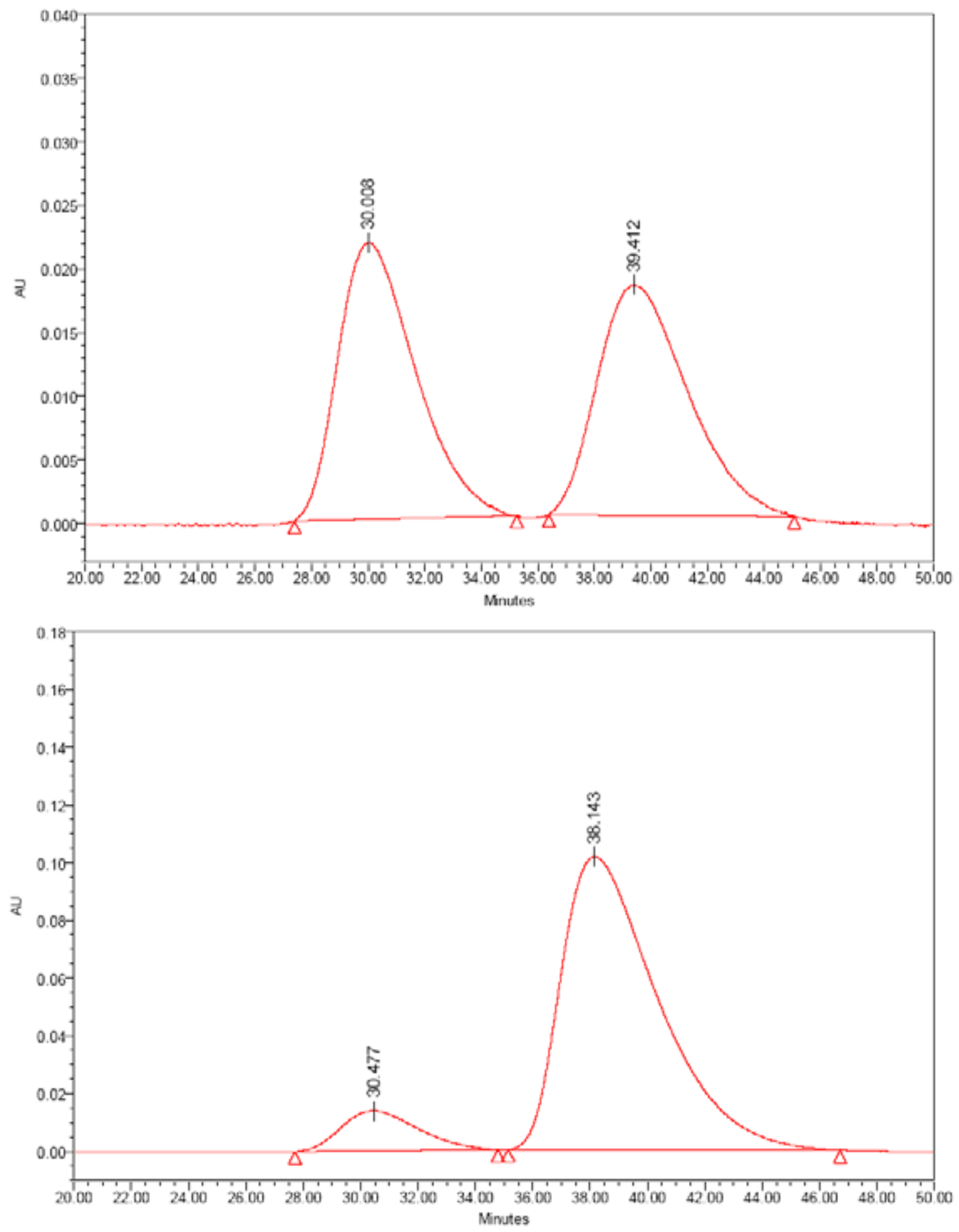
(C) Crystal Data And Structure Refinement for (S)-2f

\begin{tabular}{|c|c|}
\hline Empirical formula & $\mathrm{C}_{22} \mathrm{H}_{19} \mathrm{BrN}_{2} \mathrm{O}_{2} \mathrm{~S}$ \\
\hline Formula weight & 455.36 \\
\hline Temperature & 294(2) K \\
\hline Wavelength & $0.71073 \mathrm{~A}$ \\
\hline Crystal system, space group & Monoclinic, C2 \\
\hline \multirow[t]{3}{*}{ Unit cell dimensions } & $\mathrm{a}=18.443(8) \mathrm{A} \quad \alpha=90^{\circ}$ \\
\hline & $\mathrm{b}=8.521(3) \mathrm{A} \quad \beta=105.917(7)^{\circ}$ \\
\hline & $\mathrm{c}=27.694(12) \mathrm{A} \quad \gamma=90^{\circ}$ \\
\hline Volume & $4185(3) A^{3}$ \\
\hline Z, Calculated density & $8, \quad 1.445 \mathrm{Mg} / \mathrm{m}^{3}$ \\
\hline Absorption coefficient & $2.084 \mathrm{~mm}^{-1}$ \\
\hline $\mathrm{F}(000)$ & 1856 \\
\hline Crystal size & $0.10 \times 0.08 \times 0.04 \mathrm{~mm}$ \\
\hline Theta range for data collection & 1.53 to $25.00^{\circ}$ \\
\hline Limiting indices & $-21<=\mathrm{h}<=21,-10<=\mathrm{k}<=10,-32<=1<=18$ \\
\hline Reflections collected / unique & $10211 / 7044[\mathrm{R}(\mathrm{int})=0.0422]$ \\
\hline Completeness to theta $=25.00$ & $99.8 \%$ \\
\hline Absorption correction & Semi-empirical from equivalents \\
\hline Max. and min. transmission & 0.9213 and 0.8187 \\
\hline Refinement method & Full-matrix least-squares on $\mathrm{F}^{2}$ \\
\hline Data / restraints / parameters & 7044 / 3 / 514 \\
\hline Goodness-of-fit on $\mathrm{F}^{2}$ & 1.014 \\
\hline Final $\mathrm{R}$ indices $[\mathrm{I}>2 \sigma(\mathrm{I})$ & $\mathrm{R} 1=0.0688, \mathrm{wR} 2=0.1280$ \\
\hline $\mathrm{R}$ indices (all data) & $\mathrm{R} 1=0.1628, \mathrm{wR} 2=0.1687$ \\
\hline Absolute structure parameter & $0.026(17)$ \\
\hline Extinction coefficient & $0.00057(9)$ \\
\hline Largest diff. peak and hole & 0.348 and -0.591 e. $\mathrm{A}^{-3}$ \\
\hline
\end{tabular}


(1) Brain, E. L.; Prasad, S. R.; Thomas, C. W. Synlett 1994, 493.

(2) Zwierzak, A.; Napieraj, A. Tetrahedron 1996, 52, 8789.

(3) Hyashi, T.; Ishigedani, M. J. Am. Chem. Soc. 2000, 122, 976. 